\title{
MIKRO-ARKITEKTURE: \\ Historical Topographical Analysis
}

\author{
By: \\ Dean Mapeso
}

A thesis submitted to the Faculty of Graduate and Post Doctoral Affairs in partial fulfillment of the requirements for the degree of:

Master of Architecture

Carleton University

Ottawa, Ontario

(C) 2014

Dean Mapeso 


\begin{abstract}
This thesis looks at developing a process of designing which interacts with the various topographical layers within a site's history in order to re-construct a new narrative that re-reads into existing hidden details that are part of an established history. The process of Mikro-Arkitekture moves from a macro to a micro level of analysis to extract existing and new detailed elements from the narratives. This in turn helps to narrate moments of the history, interweaving the layers into a cohesive story that responds to a site's specific topographical history.
\end{abstract}




\section{TABLE OF CONTENTS}

A Section

Abstract

Table of Contents ii

Introduction iii-v

1 Section-1.1: History, Narratives and their details 01-06

1.2: Historical Topography $\quad 07-10$

2 Section-2.1: Scale and Proportion 11-14

2.2: Scale and Material 15-20

2.3: Fractal Patterns and Mikro-Arkitekture 21-24

3 Section-3.1: Historical Topography 25-28

3.2: Method of Narrative Analysis 29-33

4 Section-4.1: Horizons and Future Narratives 34-37

4.2: Continuation of a Past 38-39

4.3: Design $\quad 40-50$

B Section

Illustrations \& annotations $\quad 51-73$

C Section

Conclusion

74-76

D Section

Post-Script

$77-78$

E Section

Diagrams \& annotations $\quad 79-87$

F Section

Primary/Secondary Bibliography $\quad 88-90$

Image Bibliography 91-92 


\section{INTRODUCTION}

The site used to implement the process of Mikro-Arkitekture is Plaza del Diamant, located in Gracia, Barcelona. The reasoning behind choosing this as the site for my thesis comes from various experiences and influences from my time spent studying and working in the city. By spending 3 years in the Catalonian capital, I experienced first-hand many of the traditions and cultural festivities, leaving a lasting impression of the city in my memories. Living in various barrios, Gracia was one of the most influential; the ambiance, the people and the history were so profound and present that anyone entering the area for the first time could immediately feel them.

Gracia is one of the oldest districts of the city and with that comes a deep history tied to pagan worship, gothic traditions derived from the Catholic Church, modern separatist riots and bombing raids executed by Nazi Germany. When wandering through this distinct neighbourhood one begins to wonder how is such a profound history portrayed in the existing historical layers of the site. My personal experience with the specific site, Plaza del Diamant left an indelible image in my mind. Having had the pleasure of witnessing and partaking in the lifestyle and festivals that defines Gracia and the culture contained within. 
My exposure to the well-known Catalan Architect Enric Miralles came shortly after beginning my studies at a design school in Gracia. Having met two wonderful architects, Elena Rocchi and Josep Ferrando, this led to my developed interest and intrigue with Miralles and his process of capturing an essence of a design and projecting it onto a site. With the help and direction of Elena and Josep, my way of understanding design and its process began to shift.

With the help of Elena Rocchi I later began an internship at the office of EMBT - Enric Miralles/Benedetta Taligabue, I began to study and attempted to emulate his style of working, in hopes to manifest the specific quality that Enric's work is known for. Learning from Benedetta and the other fantastic architects in the office I began to develop this appreciation of the process of designing and the theme of revealing hidden elements or the essence of a project. I slowly became obsessed with the theme of patterns, repetitions and further more fractal patterns. The experiences and the interaction with these people have influenced much of my thesis work.

The historical topographical layers are narratives within a history that reference a specific moment and may be a singular or a repetitive occurrence. The process of Mikro-Arkitekture is a movement from the history to the narratives and to the details corresponding to a specific site. From this shifting of topographical layers, a change in scale occurs and the hidden details and history bring a deeper 
understanding within the human scale and everyday experience. By bringing these elements into a human scale, the project offers a tactile experience that allows users to partake in the narratives and history. The idea of MikroArkitekture is that through this process of analysis, a cohesive/harmonious project can be achieved in which the details and design elements interact with each other in a way that is topographically responding to a site's own history.

It is important to note that Mikro-Arkitekture does not follow the natural chronological timeline of an established history. Rather, it deconstructs the notion of a static timeline to reveal particular hidden details. We may know the chronological order of a particular history as told through books, paintings, movies - making it difficult to see hidden elements within. Through this movement from macro to micro and the re-organization of the narratives within a history, new and old details begin to reveal themselves. Mikro-Arkitekture thus extracts these details and gives them forms that help to physically shape the site creating a tactile environment, emotionally charging the project and allowing people to interact with it through touch, sight and sound. The research of well known historian Carlo Ginzburg on micro-history and the architectural works by the Catalan architect Enric Miralles, amongst other, help to give basis in developing this process of Mikro-Arkitekture 


\section{HISTORY, NARRATIVES \& THEIR DETAILS}

Beginning with a discussion about Mikro-Arkitekture the three main ideas that are key throughout this process, moving from the macro to micro level, are as followed:

the history the narrative the detail

The history of the site provides a general understanding of culture, heritage and society, which has influenced the development of the chosen place and its associated culture. It follows a natural chronological development and specific sequencing. The history is presented and can be told through various mediums such as writings, art, photography, film.

Narratives can vary in occurrence from a singular to a repetitive moment and can last for a short moment or span over years. They are descriptive instances that hint to the reasons why a society is, how it is, and why a culture has certain traditions. They act as a bridge between the history and the micro-details that are revealed from the narratives. Through Nigel Coates research in Narratives and Architecture, we learn that "narratives can engage with the medium of space, and form the basis on which architecture can be given meaning. ${ }^{p i}$

Looking into the specifics of what makes these narratives unique to the history can begin to answer specific questions of a site with regard to 
SECTION 1.1

culture, society and tradition. This transition from the macro to micro details, Mikro-Arkitekture looks to extrapolate these key elements, editing them to become animate objects within the overall history. This allows for the history to become physically integrated into the site and a re-reading of past events can occur, thus enhancing the connection to an already established history. In the writings of Morduant Crook, he looks at this idea of Architectural History and methods of researching this particular form of history. Crook explains that it requires a specific technique of analysis, but he emphasizes that all forms of history are concerned with the same thing: an explanation of the past. ${ }^{\mathrm{ii}}$ MikroArkitekture is a process of architecturally designing a site's history in order to bring life to its associated narratives through a process that offers a revelation of unnoticed details. Further more, Mikro-Arkitekture looks to edit a history by affixing additional informative layers in order to enhance, not to change, what are already established moments of a history.

Choosing which narratives to analyze is a conscious and deliberate act, and it is important that the selection be a result of an investigation. The designer must look for clues that reveal the embedded history, as this will influence the development of forms and the overall rendition. But how would this affect the interpretation of the site's history? And what consequences would this have on its topographical representation? 
SECTION 1.1

These narratives must stem from the same history and should never be fabricated out of non-factual evidence. This could endanger the project and lead it to become foreign not only to the site, but to the people and culture it is meant to serve. A connection to the history must be maintained to allow for a continuity and harmony to be achieved throughout the process leading up to the final design. Crook emphasizes in his research in Architecture and History, stating that: "architecture is a palpable history, culture in three-dimension it cannot be created in vacuo." iii The narratives become fixed points of reference to the history and therefore cannot be altered, however the scope of this process is not to reestablish a new chronological timeline, but to afford room for multiple narratives that can coexist and be revealed simultaneously. Mikro-Arkitekture thus looks to fill the gaps between the narratives and the structure of a history, while unveiling these details that have become lost or unconsciously overlooked. The narratives are taken and are re-organized based on a topographical layering system, breaking away from the natural sequential timeline that they follow. Coates further elaborates on a similar notion in his research stating that narrations shape and simplify events into a particular sequence, which thus begins to engage with a users imagination. ${ }^{\text {iv }}$ When the narration becomes understood the story unfolds itself as it is retold verbally, pictorially or spatially. ${ }^{\mathrm{v}}$ Furthermore, he emphasizes that a narrative or narratives shift in time, location and circumstances 
SECTION 1.1

as the dynamics of the plot unfolds along a sequential trajectory. ${ }^{\mathrm{vi}}$ But in the process of Mikro-Arkitekture, this sequential trajectory is planned by the architect investigator as he or she is in charge of the re-organization of the narratives. The chronological sequences of the topographical layers are re-learned through the act of disentangling the stories of the architectural details through the interaction with the users.

From this new historical sequence people re-establish a connection to the narrative fostered by the details that derive from the narratives of the history. People often become dependent on linking one event to another in an already established sequence to read or understand a history. This takes away from an important form of interaction between a person and his or her understanding of a history. This does not allow one to interpret what they see or how they see the history, but rather it is narrated to them in a monotonous manner removing any form of interaction one could experience with the history. Mikro-Arkitekture looks to break down this monotonous dependency on an already established historical sequence. The shift in the historical topographical layers become strengthened through the translation of the narratives from the macro to micro analysis. In his research Coates emphasizes that the physical components of a space gain significance as a direct result of the actions and experiences of the participant's 
SECTION 1.1

interaction with the architecture, who thus assembles them into a personal construct. ${ }^{\mathrm{vii}}$

A popular desk designed and built by Enric Miralles known as the Innes Table illustrates this idea of process, transformation and movement from the macro to the micro detailing. At initial glance the design appears to be that of a regular table with a unique form but the more one interacts with the table, the more it changes and begins to serve a wider range of functions. The table initiates a dialogue between itself and a person which the user remains in control of the development of the dialogue. During a discussion regarding this project, he stated that:

"The table defines different areas for working and storage, mixing together the working tools and the work itself. It responds to a concept of repetition and constant transformation: the process by which a major part of the work is to develop or weave the project into its particular time and place."viii
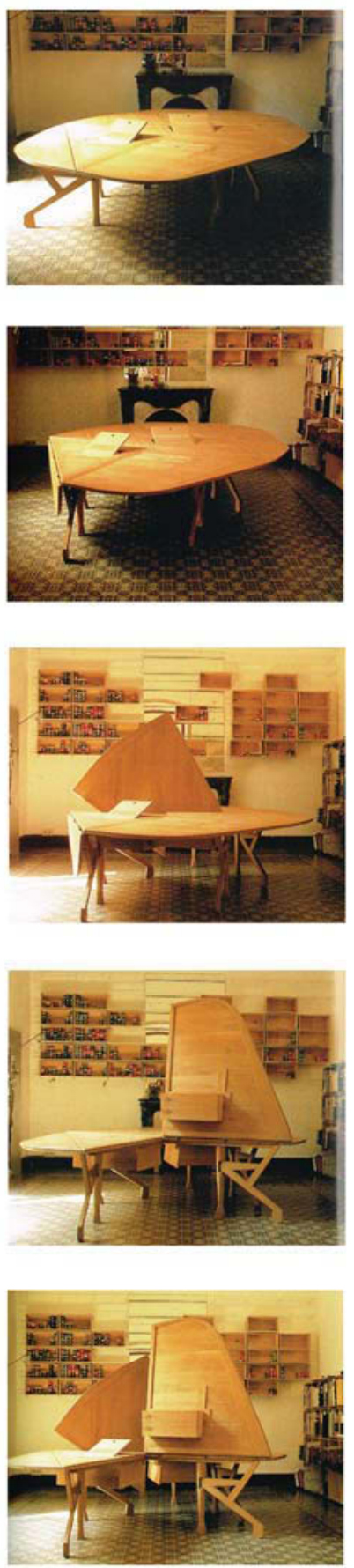

In this project it is key that the object gives a point of engagement. The history of the table is quite general and its usage is established throughout time as a place images illustrate the transformation of the table, unraveling its hidden details through each shift of its components. 
SECTION 1.1

to work, to eat or to gather. Filtering the work of Miralles through the analysis of Ginzburg and micro-history, one can begin to see an introduction of hidden details alluding to a more profound historical topography. Miralles creates a new method of interaction between a table and a user. Making the table flexible not to just a single usage but rather it responds to variety of narratives, its details animating unseen moments of the tables history. As the table folds and shifts, its method of interaction also changes adapting to the needs of the user and morphing to suite a given moment. The physical interaction between the user and table forces one to question this idea of what a table is and further more its known history. This allows the table to extend beyond its own historical limits and portray new details that are overlooked, much like Ginzburg's notion on the "normal exceptions" in a history that are often over looked but once discovered they portray a unique perspective and deeper understanding of the history being investigated.

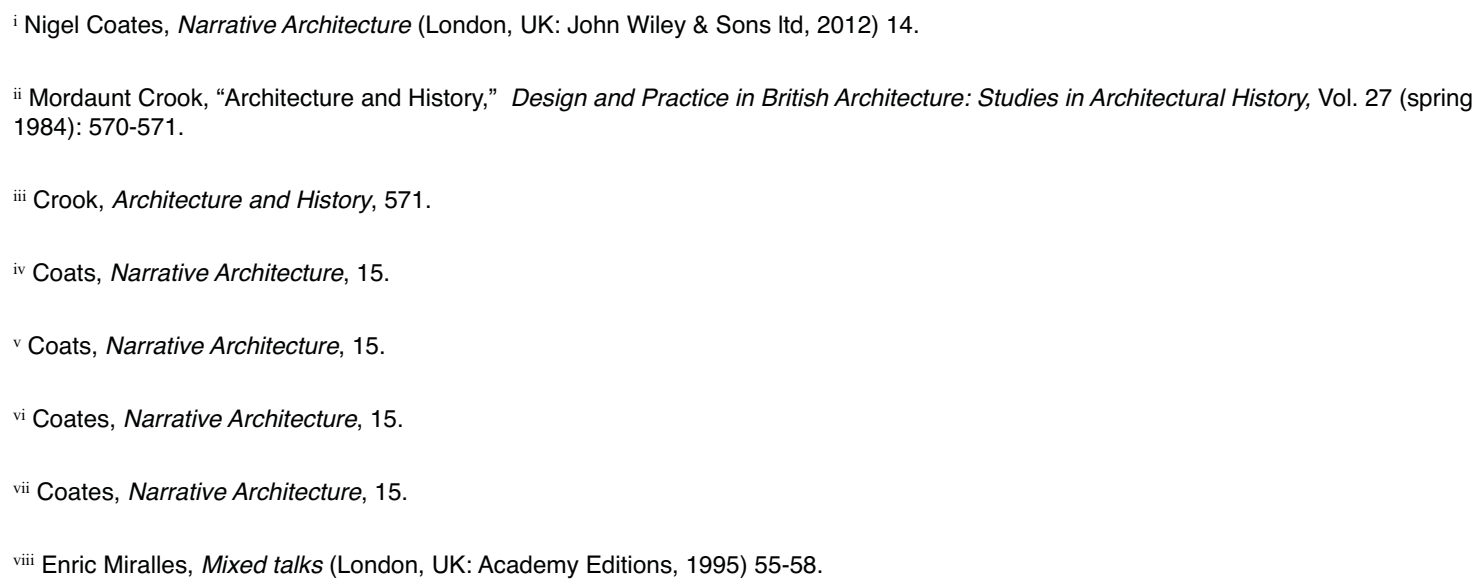


SECTION 1.2

\section{HISTORICAL TOPOGRAPHY}

Upon finding these hidden details of a narrative how does this topographically begins to develop into a historical representation of a site and its past? How does the process of Mikro-Arkitekture begin to layer details into a project in order to describe an unknown strata or untold part of the history?

Every detail is its own representation of individual layers which collectively comprise of a history. They are reflective of a series of specific narratives and moments within the larger comprehensive story of the site. The layers and details are co-dependent upon each other, each strata placed one above another in order to activate the history. Every individual strata is associated with a particular narrative that can be repeated and reconstructed and depending on the structure of how they are re-organized, this can reveal new hidden details, simultaneously building on the existing traces of the old ones. The layering sequence of the strata produces a unique detail corresponding to a particular arrangement. This part of the process can be repeated multiple times for a site and in doing so, forms new details each time. The forms generated will correspond to a specific historical topographical sequence and regardless of the chronology developed it will always relate back to the sites history, provided the narratives are factual elements of the associated history. 
SECTION 1.2

In the writings of Ginzburg, with regard to the process of researching micro-history, he highlights his experiences of investigation and analysis of historical data. With the collection of this data, he attempted to reconstruct a particular history through the selection of specific associated narratives. At this particular moment he states that: "gradually I came to realize that many events and connections of which I was totally unaware contributed to influencing the decisions that I thought I had made independently." From this notion the proposed process of Mikro-Arkitekture would allow for a distinct range of possibilities through this act of discovery. It relies on this conscious and unconscious interaction of the investigator for the collection and layering of the historical information. Peeling back the layers slowly to reveal the hidden information allows the forms to generate naturally, at the same time being guided in a general direction by the history and narratives. Returning back the writings of Coates and his notion on Narratives and Architecture he writes that: "[i]n architecture the linearity of the narrative function dissolves as the spatial dimension interferes with time." ${ }^{\text {ii }}$ Though the history, along with the narratives and details that represent it, may be more familiar to locals or those who have a knowledge of the site's past, new visitors would be able to engage with the details in a manner that allows them to establish an emotional connection to the architecture. It is through the physical interaction of the projects components and details that shifts the experience of the user. Sarah Robinson states in her 
SECTION 1.2

research that patterns of activity in the brain constantly dissolve, reform, and shift in relation to one another and that memory means that every experience absorbs something from those that have gone before and changes the quality of those that come after. ${ }^{\mathrm{iii}}$ It is through the implementation of scale and materials that help to evoke an emotional response that speaks to a memory that can be shared by many. Regardless of one's knowledge of the chronological history of a site, Mikro-Arkitekture aims at perpetuating a singular or multiple emotional connections that can be identified universally. If we are to close our eyes, take a deep breath and summon meaningful memories, we quickly notice they are tied to a specific place. This place evokes a network of sensations, the warmth of sunlight, particular smells or sounds, people. Architecture creates a place for the accumulation of ephemeral and tangible memories to become manifested through tangible objects which bring them to memory and shape new memories. ${ }^{\text {iv }}$

By giving people a general basis of emotional experience this allows a wide number of users to simultaneously partake in rituals encouraged by the architecture while being propelled deeper into the details, its associated narratives and history. Breaking away from this chronological history allows for the extraction of these hidden elements, thus the project can be easily integrated and adapted back into the physical site and its historical topography. The project and its elements do not feel foreign or alien because they have originated from 
SECTION 1.2

this singular point of reference, helping to give the design value to the culture and society it serves. Carlo Ginzburg, in his writings on history and its portrayal through writing and cinema, states that starting from the same premises (history), it is possible to arrive at a similar conclusions independently. ${ }^{\mathrm{v}}$

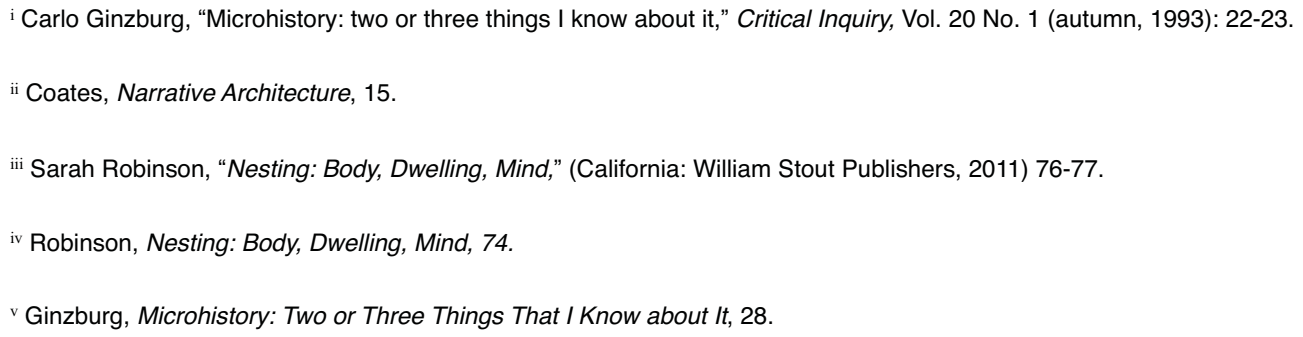




\section{SCALE \& PROPORTION}

Topographical layers act in relation with each other to animate the hidden details that lie between new and existing narratives. There is a constant interaction between the actions of concealing and revealing. But what drives this act of discovery? How would the scale of the details affect their ability to retain and re-tell a narrative in connection to a history? And how do they act as a catalyst for rituals to occur within a site, activating the history and allowing it to become a tangible interactive object? The question of scale helps to understand better central ideas about Mikro-Arkitekture and the manner in which it functions.

The reading of the narratives is a conscious act by the designer that leads to the rediscovery and the analysis moving from a macro to a micro level to unearth details that are hidden. In the writings of Ginzburg on the topic of microhistory he alludes to this idea of analysis in relation to scale stating that certain phenomena can only be grasped by means of a microscopic perspective. ${ }^{i}$ As mentioned previously these narratives are never fabricated and should derive from the embedded history. This maintains continuity and allows for a connection between the narratives, although the chronological path is disassembled and rearranged, they still retain a cohesive emotional and psychological language that 


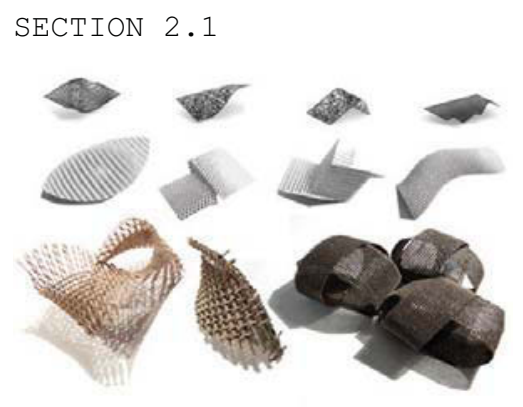

ties back to the singular origin point in the history. There is a gradual movement leading from the history to its details and this harmony should be maintained in order to not create an abrupt interruption from this transition from a macro to a micro-analysis.

The manifested details should be

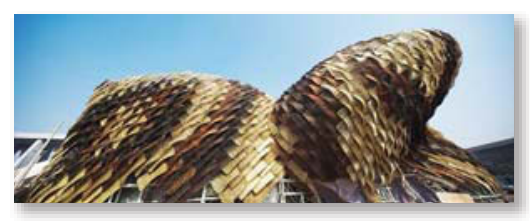
designed with a sense of the human scale. This notion can be analyzed in the

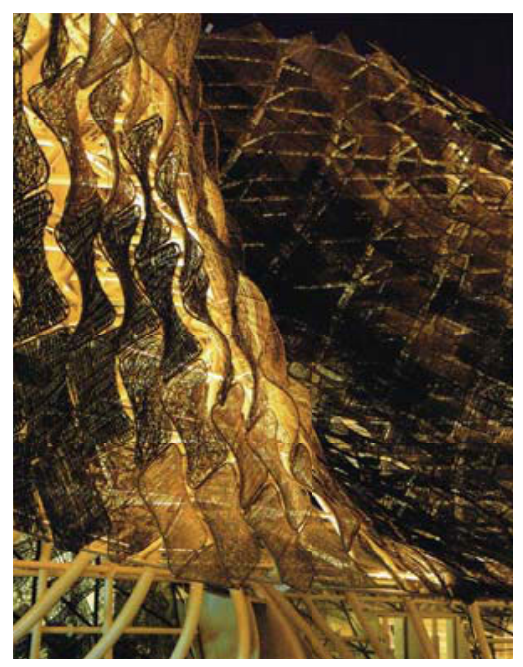
process of working of Enric Miralles and his ideas about manual work, which entails experimenting with different materials through modelling to define the expressive qualities of a project. The idea of working with materials by hand allows for an intuitive development of the materials and how they can be shaped to suit the function of the concept. The human body should narrate the relationship between the 
SECTION 2.1

scale and the proportion of each detail in relation to one another. If the details take on a larger scale and extend beyond the limits of the human body, a disconnection occurs. If the objects grow out of our ability to interact with them, both physically and emotionally, then the details become these inanimate devices loosing the ability to re-tell the narratives and stunting the experience for the people who inhabit or visit the site.

This notion of scale and proportion plays a vital role in how we perceive and interact with a project. Lydia Haack and John Hopfner highlight in their studies on space optimization that "[t]he reduction in [scale] of the object is associated with an increase in its sensory quality and symbolizes art and harmony between man and
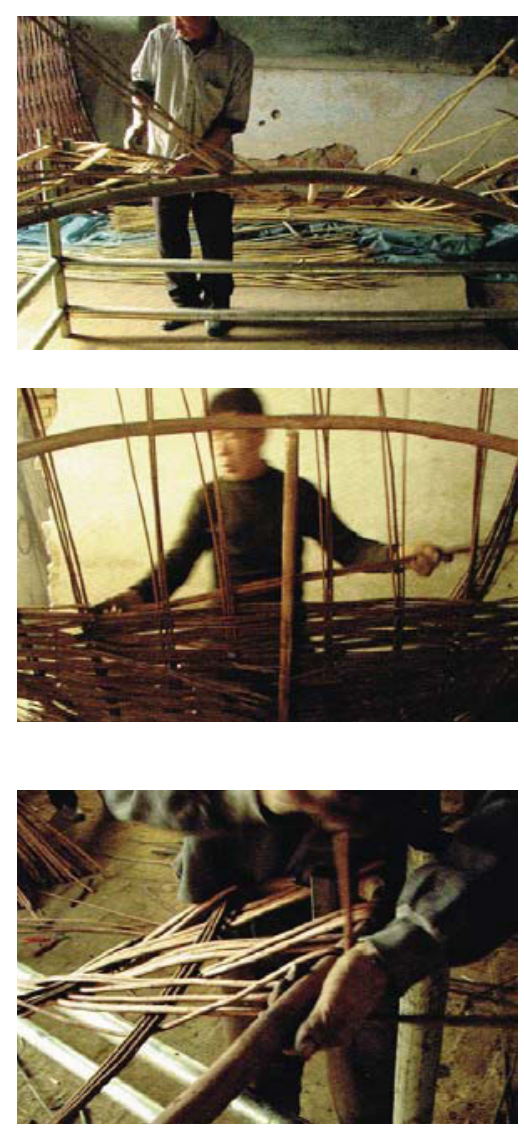
SECTION 2.1

nature." $\mathrm{ii}$ By producing a detail within the limits of the human body there is an embedded tactile quality that comes out and instinctively creates a unique bound between the user, the history and the details. The scale acts as a stimulus for the people to engage with the site's history; this physical interaction justifies the complexity of the historical topographical layering.

\footnotetext{
${ }^{\mathrm{i}}$ Ginzburg, Microhistory: Two of Three Things That I Know about It, 27.

ii John Hopfner, "Microarchitecture - experiments in space optimisation," in Small Structures: compact dwellings, temporary structures, room modules (Germany: Edition Detail, 2010) 11.
} 


\section{SCALE \& MATERIALS}

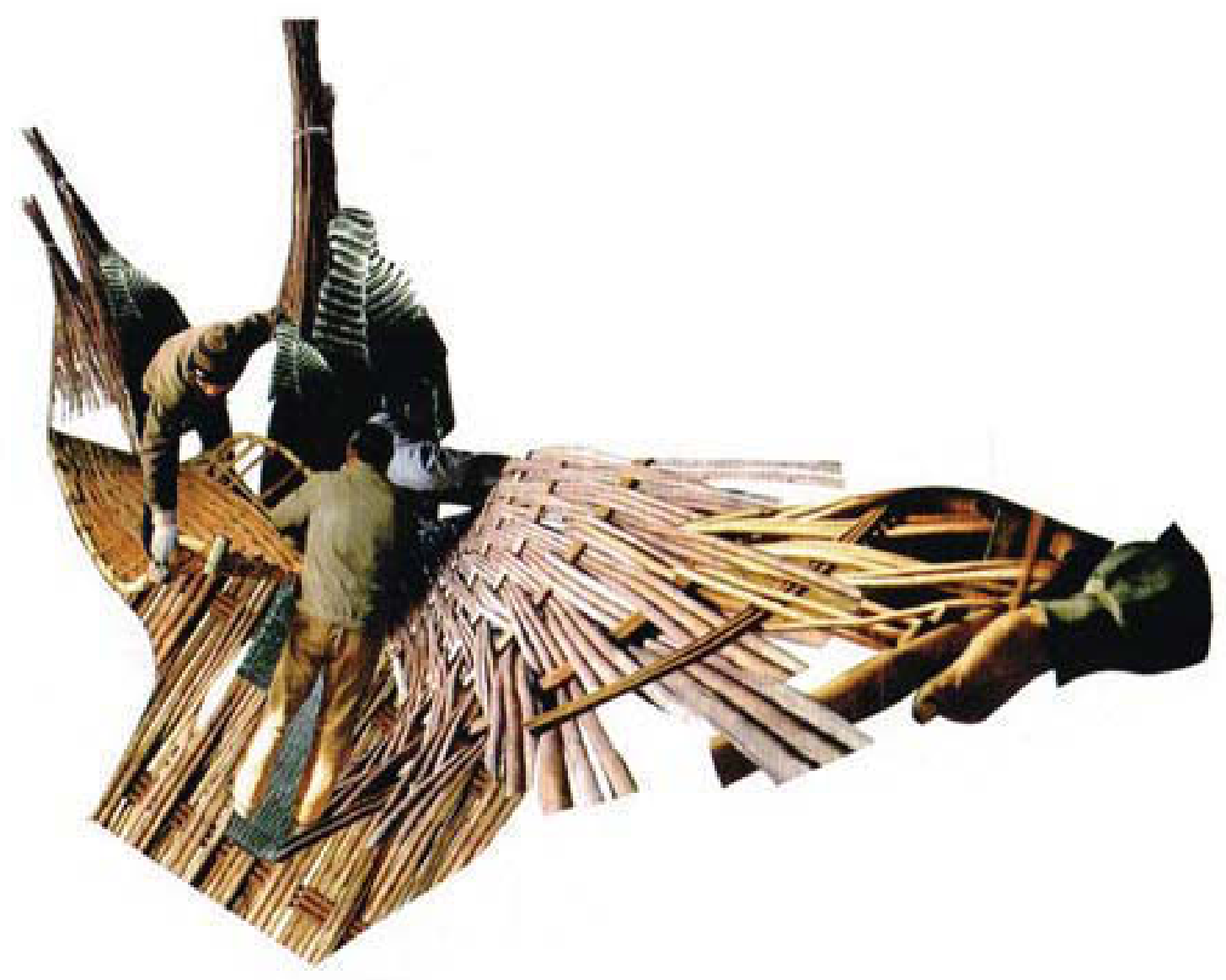

6. Collage - Spanish Pavilion 2010: this collage captures the projects process and essence through the use of the wicker material. It illustrates this notion of experimentation and exploration of a material in order to achieve a
greater design. 
Dwelling further into this theme of scale, Mikro-Arkitekture and the elements derived from this process are not necessarily constrained by a size limit but rather this process can be easily adapted into any scale and any site. Materials play a vital role in our sensory interaction with a project, while the size of an object would question our physical capability to interact with it, the materials used should be scaled in accordance with the required sense of tactility necessary for the project. The act of scaling a detail from a macro to a micro level and vice versa is not solely based on this notion of physical dimensioning but can also be a scaling of the actual materials. In the work of Thomas Schropfer on the theme of Material Design he looks at the idea of how materiality informs the design and development of architecture. More specifically in the chapter titled Inherent Expression: A Greater Understanding of Materials' Potential for Expression, he states: "a scale-less investigation into the qualities that define materials, both physically and conceptually, leads to a sophistication of their use within a greater design concept." ${ }^{\text {i }}$ Whether it is the physical scaling or material scaling of a detail, the result is an object that has an increased ability to engage with the users, thus activating the rituals that can or will occur on the site. The scale in which these objects interact with the site should be in proportion to each other. In the projects of Miralles he has the unique ability in playing with various elements of a project while maintaing this ideal proportion or scale that suits the site, project and 
materials. This is illustrated in figure 7, depicting details of the Icaria Pergola designed for the Olympic Village, a series of these pergolas were constructed adorning the central walkway. These pergolas were inspired by festivals and parades that occurred during the summer months in Barcelona.

"Perhaps the best means of approaching this work - without forgetting that the procession is animated only because of the people who are hidden beneath its elements would be to concentrate on the shadows and fragments to observe how the shadows fall on the ground so far away from the structures, from the point of origination, while the structures themselves grow tall from the point of contact with the ground. The projected shadows are like the footprints of the pillars: they mark the rhythm of the promenade and the passing of people. The wooden elements have the same proportions as interior furniture[.]"ii
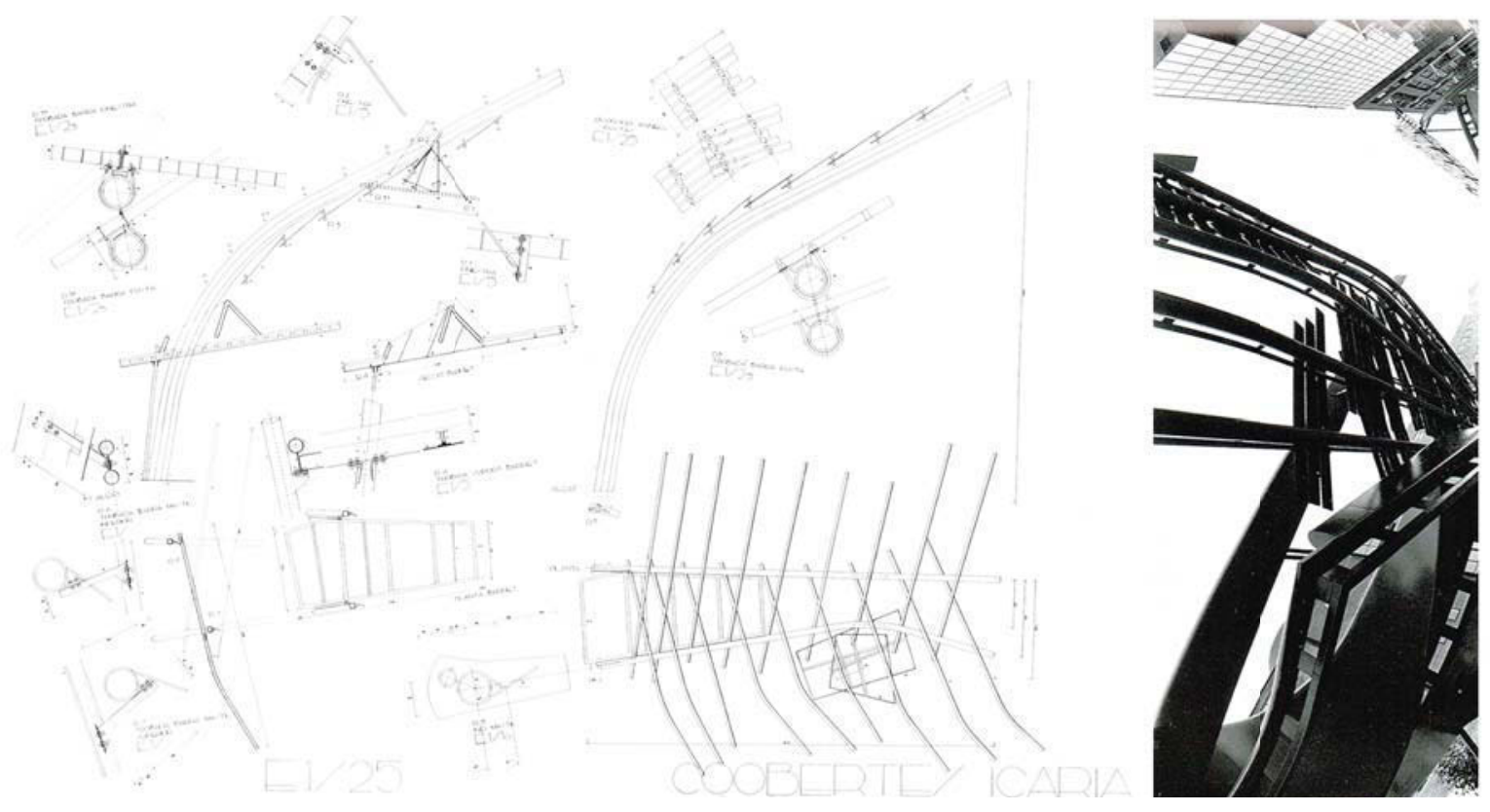
SECTION 2.2
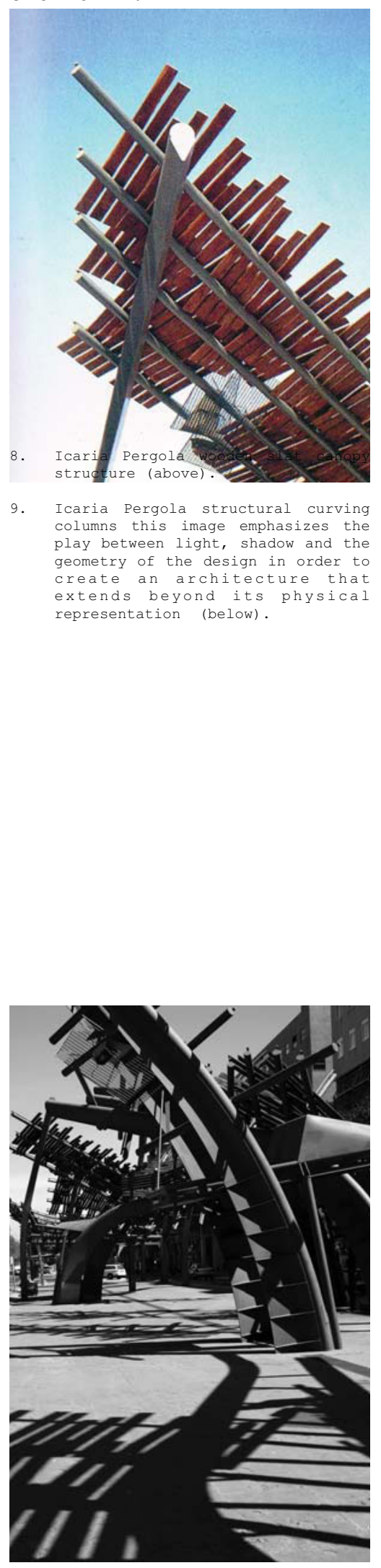

Miralles takes the details from the narratives and creates a story through the use of volumes and geometries, allowing visitors to re-live moments of the festival. He uses materials that reflect specific moments of the narratives that are never seen by the people which functions on various planes to activate the design. This notion is further strengthened once again by Ginzburg and this discovery of abnormalities in a history. These abnormalities reveal a more profound understanding of the associated history. The use of materials throughout his projects is done in such a way that generates a dynamic interaction between form, scale and material. This is energized by the introduction of light and shadows into the design of the project. Miralles uses the play between 
SECTION 2.2

the two elements to negotiate the juxtaposition of the different components and details of the design. The pergolas are dependent on the use of lighting in order to reveal hidden moments of the narrative that are strategically embedded into the architecture, these moments reveal themselves in the forms of shadows. Miralles design the components of the project to a more human scale but the play between lights and shadows allow the architecture to grow. The structure itself remains static but the shadows and light animate and breath life into the architecture. His architecture works on these various planes where he carefully articulates the essence of a project through the projection of shadows.
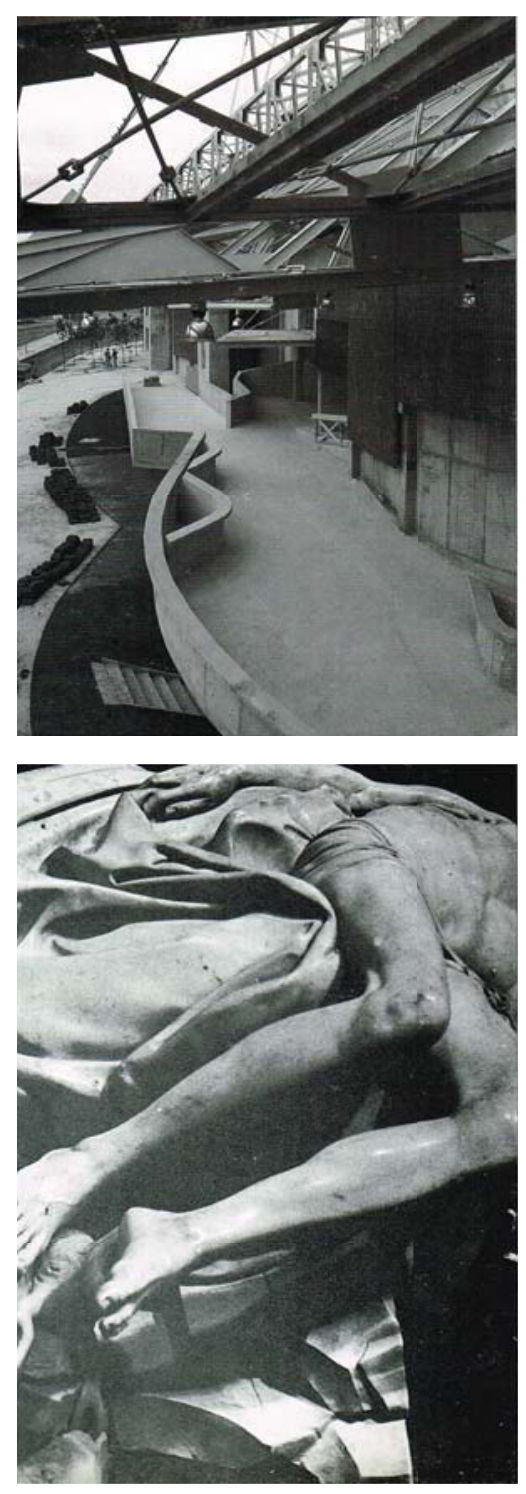

"The movement of the ramps precisely locates the access on the long facade, which is lined by the vertical supports of the roof. It reminds me of the famous Pieta sculptures, in which the body of Christ is stretched out. The building lets us see the articulation of its surfaces and its sprawled limbs. In the act of allowing its side to be vulnerable, it becomes public." 
SECTION 2.2

The process of Mikro-Arkitekture works in a similar fashion but rather than using light and shadows, it uses the system of historical topographical layering of narratives in order to cast these "shadows"and thus extract the details that can be developed into physical objects speaking to this juxtaposition of the topographical layers. A narrative develops between the different components that interact in a harmonious manner whether they are furniture, construction details or plans. There is a relation between the final project and the drawings produced emphasizing this shift between concept and drawings to the built project while maintaining this idea of scale in relation to the materials.

\footnotetext{
i Thomas Schropfer, Material Design: Informing Architecture by Materiality, 25.

ii Enric Miralles, Mixed Talks, 19.
} 
SECTION 2.3

\section{FRACTAL PATTERNS \& MIKRO-ARKITEKTURE}
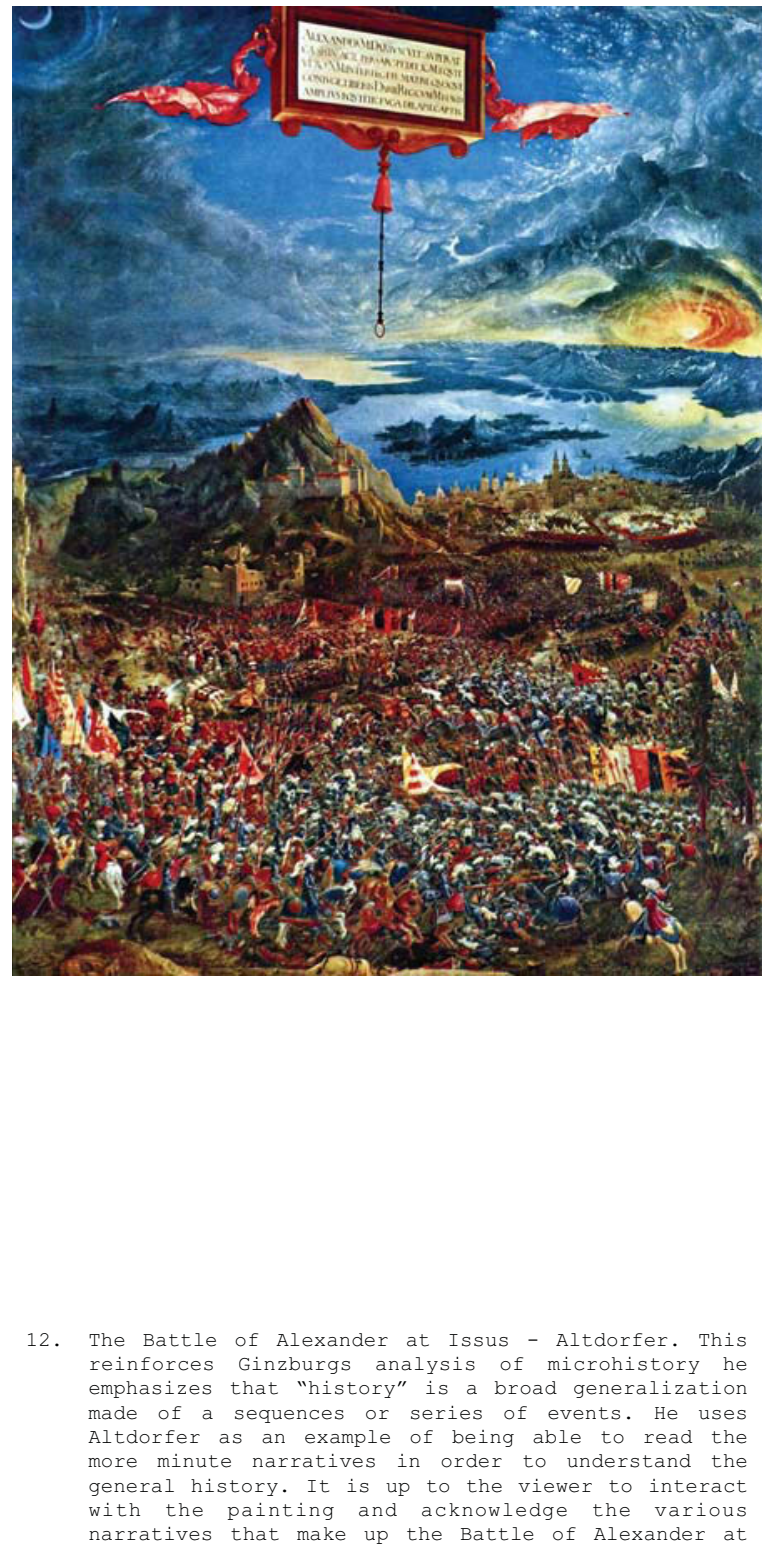
In his research on micro-history Ginzburg alludes to this idea of scale and patterns that is created over the development of a history. He looks at the work of a German Renaissance painter and engraver named Altdorfer and his ability to abstract depictions of a narrative and reveal details, Ginzburg writes that: "[a] close-up look permits us to grasp what eludes a comprehensive viewing and vice versa." ${ }^{\prime}$ The level at which these details are developed can be further explained by this notion of fractal patterns. When viewing a fractal pattern at a micro level one sees only a small part of the fractal and when the view embraces a macro level, a larger part of the pattern is revealed but the detailed subtleties are lost. It is only through this act of moving from a macro
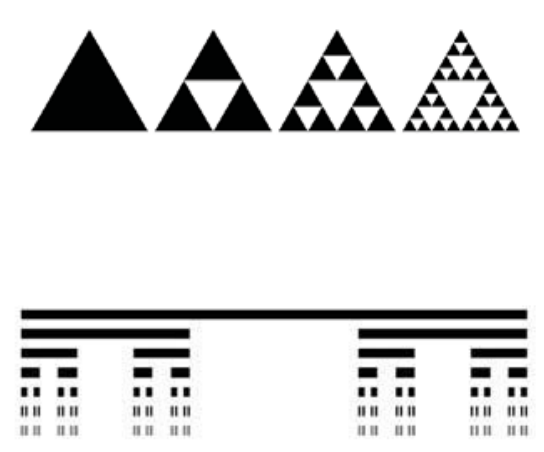

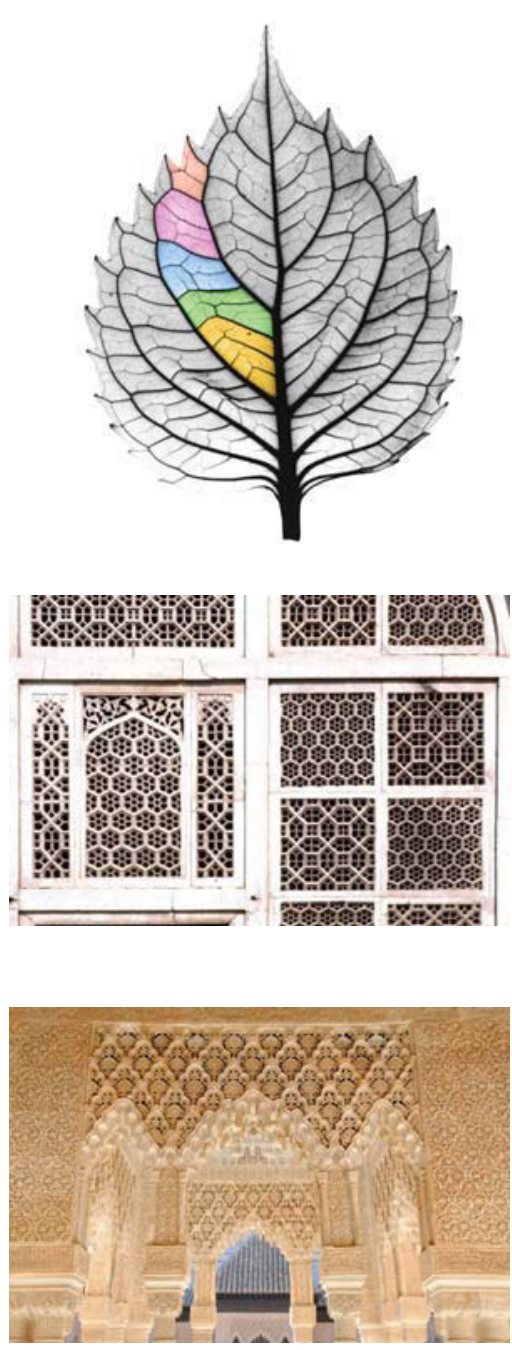

15. Example of natural fractal patterns in a leaf (above).

16. Alhambra, Granada, Spain Fractal patterns used in architecture in an attempt to mimic nature. These type of motifs were very common throughout middle eastern architecture (middle).

17. Alhambra, Granada, Spain Honeycomb styled portico entrance (below). to a micro level and vice versa that these details and specifics of a fractal pattern become evident. This movement is constant and is necessary to fully understand the relationship between the details and the history. The narratives act as this connector between the history and the details, where each part is dependent on one another in order to complete the pattern. Much like a fractal pattern, if it is purposely altered in any manner than the pattern cannot be completed, similar in fashion to how a narrative can never be fictitious. The idea that a fractal can never end can be seen in relation to history itself as an ongoing infinite string of narratives which continuously develop over time. In the work of Carl Bovill titled Fractal Geometry in Architecture and Design, he writes that: "[t]he fractal dimension can 
SECTION 2.3

be related to information theory as a measure of mix between order and surprise. The higher the fractal dimension, the more surprise is mixed into the underlying order." ${ }^{\text {ii }}$ By analyzing the topographical layers embedded in a site's history, one begins to reveal hidden details that help shape the visual representation of the architecture. The history, in comparison to fractal patterns, is the broader or macro analysis, while the narratives are its highlighted sections that offer clues or suggestions as points of investigation while the details speak to the specifics narratives.

\footnotetext{
i Carlo Ginzburg, "Microhistory: two or three things I know about it," 26.

ii Carl Bovill, Fractal Geometry in architecture and design (Boston: Birkhauser, 1996) 116.
} 
SECTION 3.1

\section{HISTORICAL TOPOGRAPHICAL LAYERING}

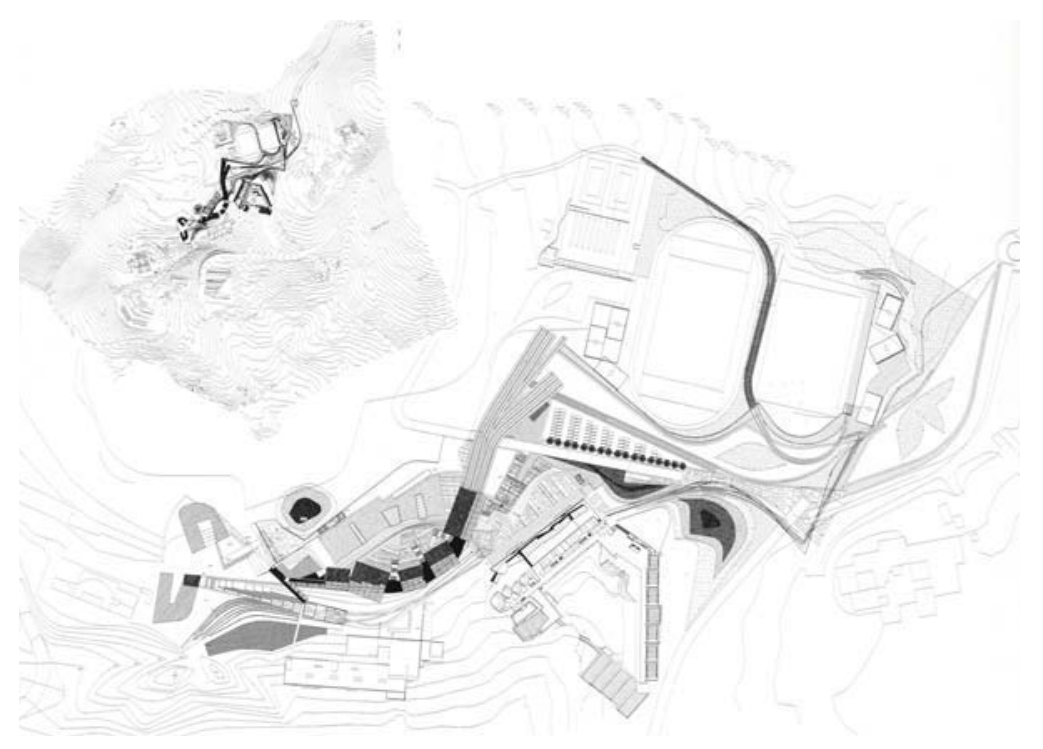

18. Site plan of the Campus of Vigo University 
These topographical layers create a landscape that is reflective of a section through the historical and narrative strata of the site. Together the layers create a historical topography that is activated through human interaction. In the writings of David Leatherbarrow titled Uncommon Ground he researches what he considers to be two topics central to architectural work: the construction and siting of buildings. He analyzes this notion of a 'horizon,' which he does not view in a conventional manner. He first describes the horizon as the extreme limit of the surrounding landscape. ${ }^{\mathrm{i}}$ The horizon is always a potential site, its remote constancy reinforces the sense one has of the radical contingency of human experience. ${ }^{\text {ii }}$ The horizon for Leatherbarrow is not the conventional site line of where the ground meets the sky. Rather it moves vertically and horizontally but the difficulty in grasping and understanding a horizon of a site is also discovering its hidden stratification. ${ }^{\text {iii }}$

The layering of the topography provides a new means of interpreting the site's history but it is also important in understanding the stratification of the layers and how moments of the narratives, in section, link with each other. Anatxu Zabalbeascoa, referring to the work of Miralles, states that his projects are architecture of the land that involve a humanization of the program and appreciation of the topography, meaning, the visible, physical land as well as the 


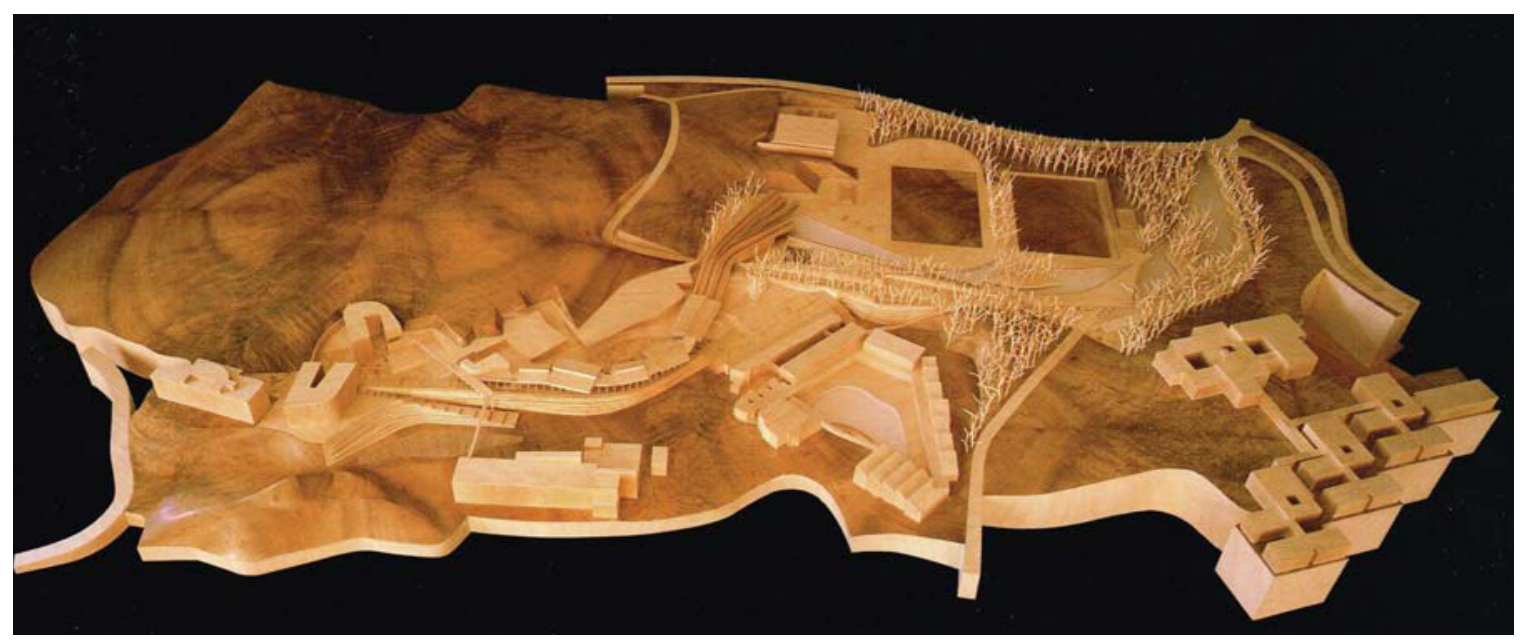

19. Hand model of Vigo University Campus the line between building and landscape become blurred in order to emphasize a relation to the contours of both site and building

memories contained within it. ${ }^{\text {iv }}$ Miralle's work is a way to "transform [a site] by means of a major topographic change"v, more specifically in reference to one of Miralles's projects the University Campus of Vigo, he further elaborates that "it is difficult to separate public space from construction ... a square and a building are the same thing."vi Analyzing the site model and the site plan the distinction between the physical landscape/building are blurred, forms are developed by following the physical contours. Mikro-Arkitekture uses a similar process but instead of analyzing the physical topography, a shift in focus is placed onto the historical contours and strata. Investigating the layered strata assists in establishing a three-dimensional image of the contours. Highlighting a section of intrigue focuses the analysis into a specific development of details that correspond to a particular moment within the narrative. This moment thus 
SECTION 3.1
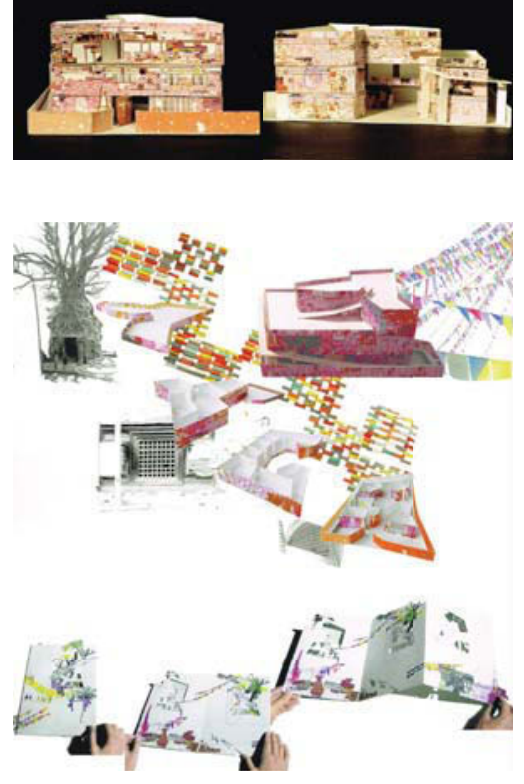

20. Primary School - Kathmandu, Nepal (2005). This illustrates the translation of the physical contours of the site and how through the technique of hand modelling and working within a human scale the project begins to shape itself from this intuitive process. MikroArkitekture prork in Mikrofashion using the historical topography to generate forms through a process of rediscovery and re-telling. transcends the multiple strata's carrying added contours from the other layers before emerging as a new form.

i David Leatherbarrow, “Uncommon Ground,” (Massachusetts: Massachuetts Institute of Technology, 2000) 170.

ii Leatherbarrow, Uncommon Ground, 172.

iii Leatherbarrow, Uncommon Ground, 173.

iv Anatxu Zabalbeascoa, Igualada Cemetery, 10.

V Benedetta Tagliabue, EMBT: Work in Progress, (Barcelona: ACTAR D, 2006) 88

vi Tagliabue, EMBT: Work in Progress, 88. 
SECTION 3.2

EMOTIONAL \& TACTILE ELEMENTS
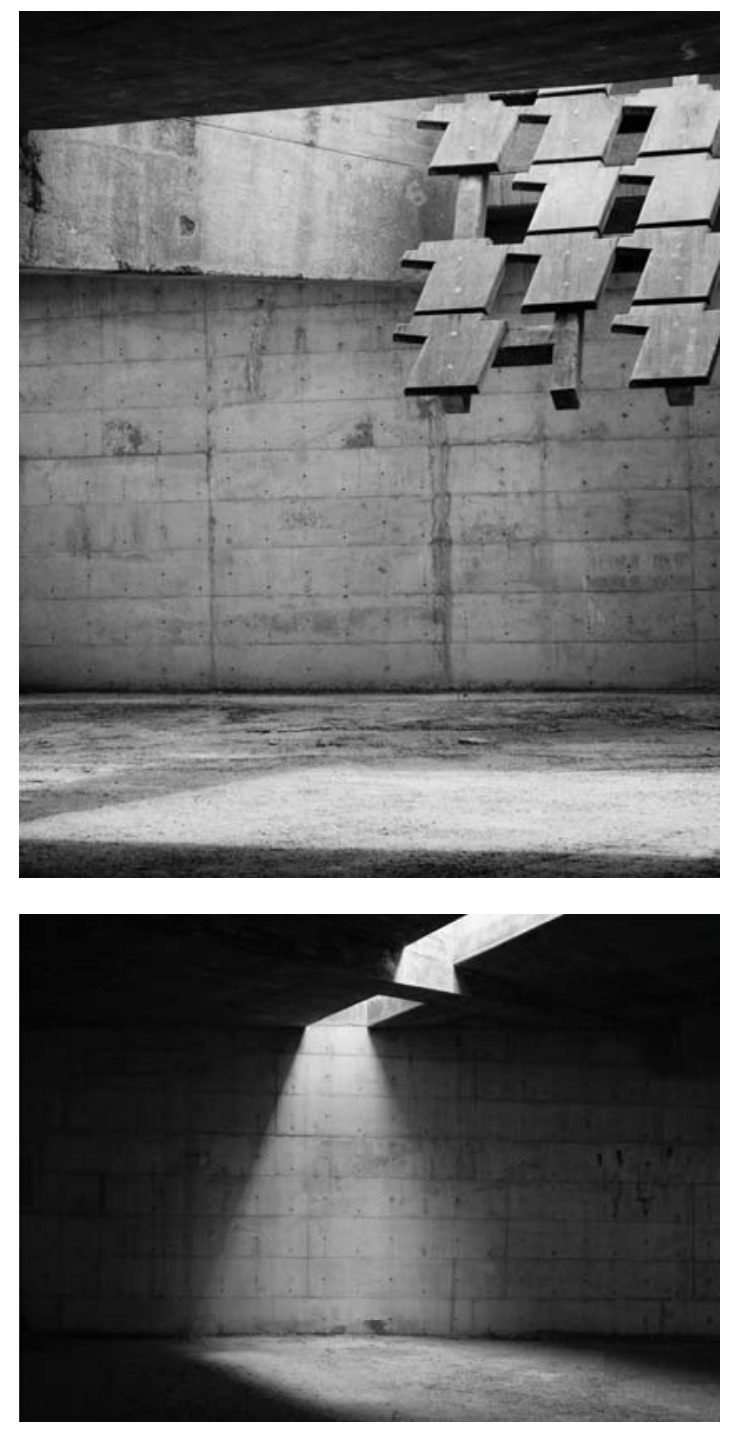

21. Igualada Cemetery - EMBT: main passage way into the cemetery. Depicts roofing structure with cantilevered components that allow light to enter which they are decorated with a textile pattern. The cantilevers, tiles and structure is made all of formed concrete.

22. Igualade Cemetery, Catalunya, Spain by EMBT main passage way into the cemetery. Specifically planned pockets of light allows a contrast between the heavy material and emotions. 
A natural complexity begins to develop where elevation, section and plan begin to merge and this hybrid project starts to take on new forms. It is important to maintain throughout this process this idea of scale when moving from the macro to the micro level. The scale at which each detail, layer and element is represented plays a vital role in its perception and interaction. Much like the work of Miralles there is a tactile quality of the project, regardless of the overall size of the product, because the details are done in such a way that they can be understood in relation to the human body. In the writings of Gerhard Kolhofer and his research on the topic of mobile architecture more specifically how the human scale could enhance a users understanding of a space. He states that:

"The possibility of directly experiencing a building through its human scale increases the likelihood that its complexity will be perceived and understood. This creates a much closer attachment by the user than with large buildings, an attachment that is frequently reinforced by interactive elements. A person who can change his environment through his interaction with the building or can set it in motion loses the traditional respect for perfectly designed architecture and a direct relationship is more easily formed."

Mikro-Arkitecture and its products are not defined by size, this process can be easily adapted into any scale and site, but rather they are expressed through the complexity of the interaction between the historical topographical layers. Through this interaction the development of details can be shown and moments of the narratives are revealed to the visitors who begin to be engage with the topographical history in a tactile and emotional way. Looking at the Scottish 


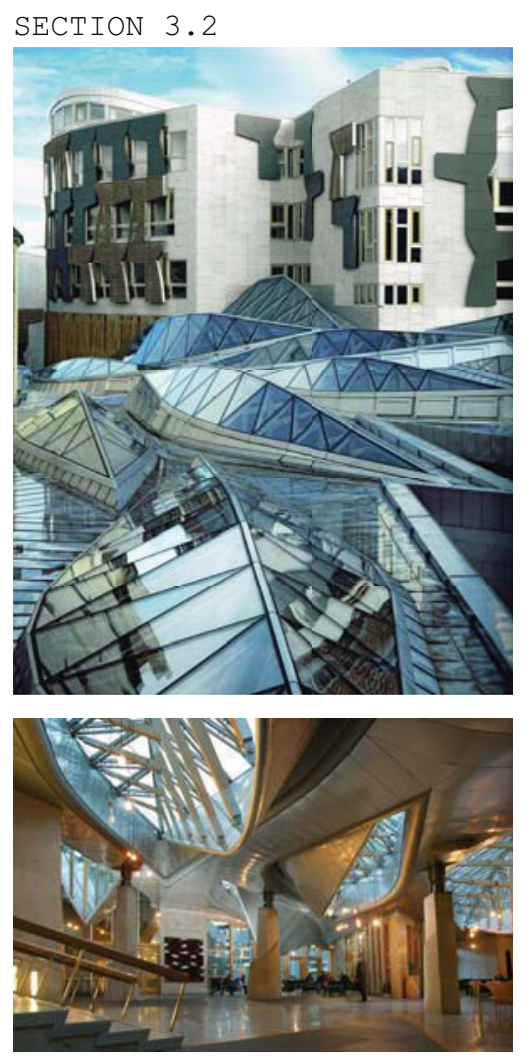

Parliament, a project completed by EMBT in 2009, Benedetta Tagliabue points out that the building opens its public space to the general concept of the Scottish landscape and that "it is not difficult to imagine pensive walks outside of the building, with thoughts running through one's mind ... seeking the help of a lonely walk during a reflective moment." ${ }^{\text {ii }}$ Adding emotional elements into the architecture mixed with that of history allows for the development of a project to have a more profound meaning to the site.

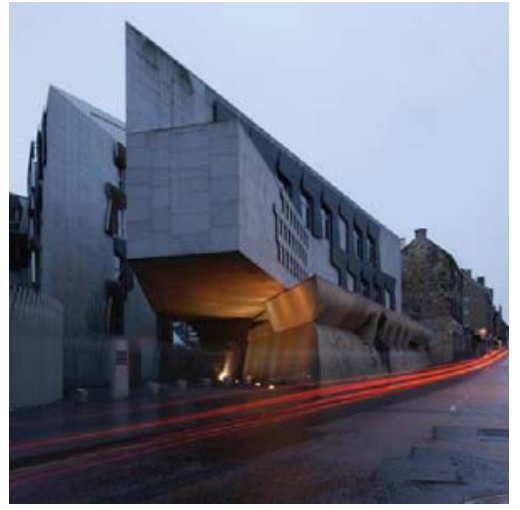

Architecture is a mixture of structural techniques, aesthetic instincts, and traditions, but history adds a fourth dimension to the three-dimensional language of architecture. iii By adding this element of history to the process it assists in activating the architecture. 
SECTION 3.2

In turn, this creates an association to an already established chronological story allowing it to become part of a future history. By providing these moments of discovery within the project this gives a greater meaning and purpose to the architecture. With regard to English Gardens, Coates describes this technique of objets trouves, which are purposely planted moments in a garden that could be re-discovered by visitors whenever they would stroll through the garden. ${ }^{\text {iv }}$ Various objects were scattered across the scene to create reference to a general history, specifically one of antiquity. But since they are never visible simultaneously, and even though they are fixed in space, they succeed in evoking a sense of the unique moment. ${ }^{\mathrm{v}}$ The process of Mikro-Arkitekture looks to do the same but instead of these objets

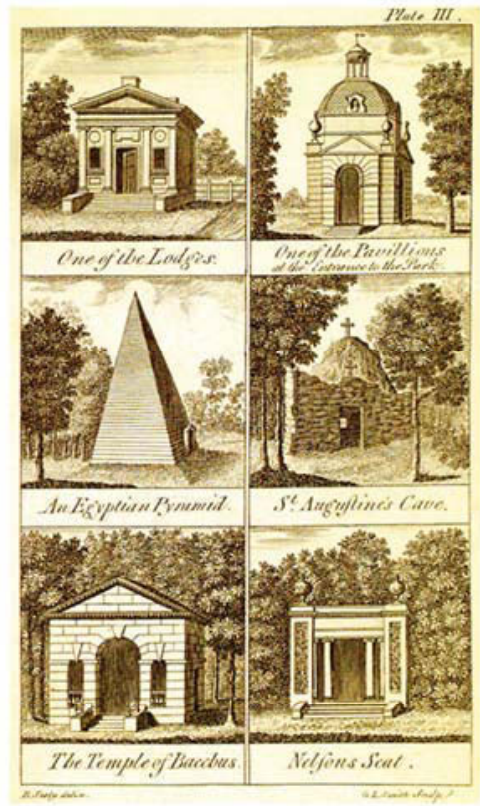


SECTION 3.2

trouves, details are produced and planted into the site which work harmoniously to re-tell a set of narratives that are re-organized from their chronological construct into a new sequence.

\footnotetext{
i Gerhard Kalhofer, "Mobile Architecture," in Small Structures: compact dwellings, temporary structures, room modules (Germany: Edition Detail, 2010) 39.

ii Bendetta Tagliabue, EMBT: work in progress, 123.

iii Mordaunt, Architecture and History, 571.

iv Coates, Narrative Architecture, 21.

v Coates, Narrative Architecture, 21.
} 
SECTION 4.1

\section{THE HORIZON OF FUTURE NARRATIVES}
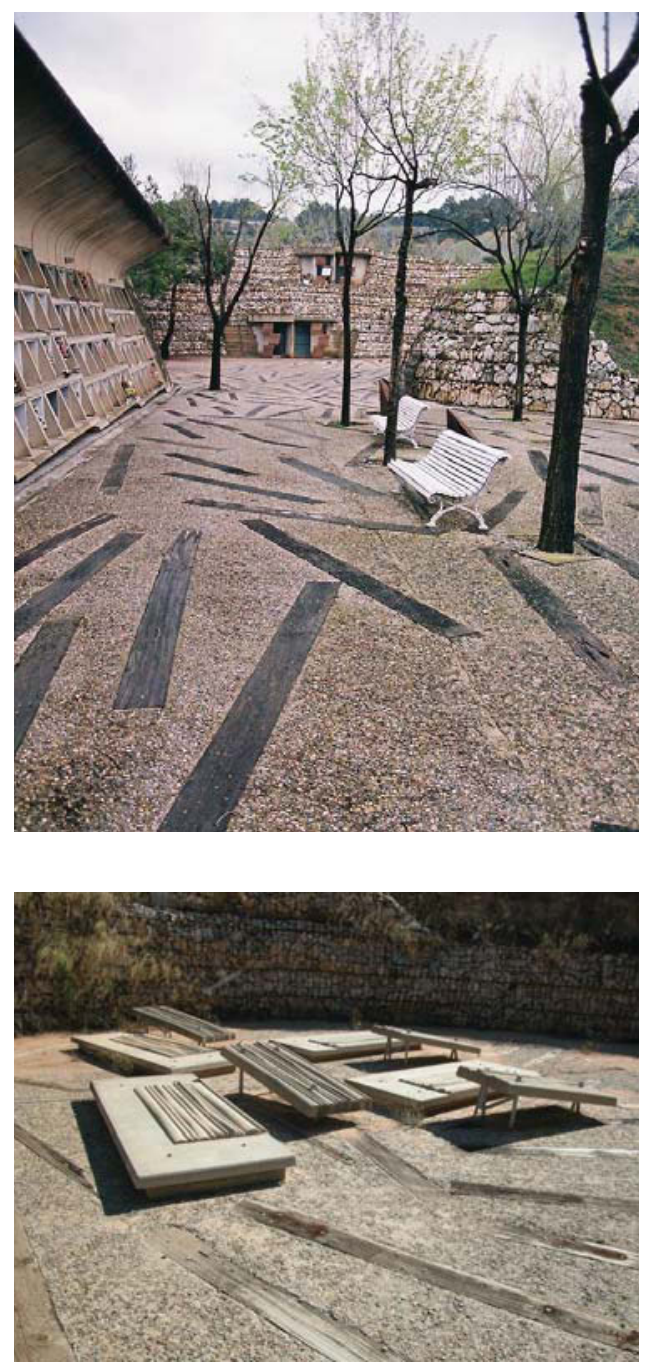

\footnotetext{
27. Igualada Cemetery - EMBT:processional walk

28. Detail of Igualada Cemetery
} 
Looking at the relationship between the history and what is revealed takes on this complexity throughout this process imparting onto each detail a character. Although these elements work to create a harmonious design each individual component is representative of a narrative and the materials used should speak to their individual uniqueness while at the same time representing the history as a complete and singular architectural project. Looking at the cemetery project of Igualada by Miralles and Pinos, Anatxu Zabalbeascoa states that the materials used in the cemetery's construction help to articulate the meaning of the work as a whole. ${ }^{i}$ As stated previously the details encourage a specific emotional response that is echoed throughout the various components developed from the analysis of the historical topographical layers. This in turn allows the project to develop over time into its own proper narrative, while a connection is maintained to the history and acts as a catalyst for people to participate in rituals at site. Sarah Robinson states in her work that: "[o]ur feelings about a particular place may be personal, but the feelings grow out of collective experiences that do not occur elsewhere. They are specific to and belong to the place." ${ }^{\text {ii }}$ The architecture developed from this process looks to enhance the experience of the site by paralleling this emotional experience of the individual users with that of the narratives, it is through this connection that the project finds grounds generating a basis for development into future narratives. 
This notion of site analysis and investigation has always been present as a beginning point for any design project. We learn through Crook's research in Architecture and History that methods and differences between architectural history and History is the nature of the evidence with which it deals with and the techniques specifically developed to assimilate that evidence. ${ }^{\text {iii }}$ It is through the process of historical architectural topography and analysis of the macro to microdetail that allows for Mikro-Arkiteture to break away from standardized construction modules, techniques and materials in order to generate a project that better responds to a site specifics - cultural, traditional, or sociological conditions. Much like Ginzburg's search for the "normative exceptive" in a history that provide hidden information of a history, Mikro-Arkitekture looks to develop specific details that capture and reflect the unseen moments of a narrative and the history. Leatherbarrow highlights in his research on materials with regard to the work of Le Corbusier, Wright, Mies ven der Rohe and Richard Neutra that:

"Forgetting for a minute what these architects wrote, looking carefully at their ways of working with the city, one can discover that their projects accepted a double constraint: acknowledging what remained relevant from the past and imagining what would be required for the future because circumstances would have changed."iv

What Mikro-Arkitekture attempts to achieve is a design that responds not only to a history but that is also able to adapt to future narratives. On the theme of terrain and mental mapping, Coates highlights that through use of narratives and 
SECTION 4.1

mental maps, which he refers to as derive, architecture should look beyond a static set of uses and look at what already exists in order to understand how to project towards the future. ${ }^{\mathrm{v}}$ Returning back to Leatherbarrow's ideas about horizon and topography he makes it clear that horizons are things not seen and absconded or recondite. This gives horizons this capability to be what might yet occur while representing what has happened in the past regardless of how it looks in the present. ${ }^{\mathrm{vi}} \mathrm{A}$ building is always incomplete, it is a form that is never finished or final, and is completed as time goes by. ${ }^{\text {vii }}$ These unique details characterized by specifically chosen materials activate the architecture and engage it with the history and the people, allowing it to become flexible in its ability to adapt to various moments of a specific site.

\footnotetext{
${ }^{\mathrm{i}}$ Anatxu Zalbabeascoa, Igualada Cemetery, 21.

ii Sarah Robinson, Nesting: Body, Dwelling, Mind, 141.

iii Morduant Crook, Architecture and History, 571.

iv David Leatherborrow, Architecture Oriented Othersiwe, 73.

v Nigel Coates, Narrative Architecture, 34.

vi David Leatherbarrow, Uncommon Ground, 202.

vii Leatherbarrow, Uncommon Ground, 202.
} 
SECTION 4.2

\section{A CONTINUATION OF THE PAST}

The unseen and hidden elements is what gives rise to all the adjustable instruments that animate a building, as it gives rise to what the building is able to narrate. ${ }^{i}$ It is this exact notion that is key in making Mikro-Arkitekture successful in its attempt to be a fully integrated part of a site's history. The manner in which these details are manifested and the materials used to illustrate them define the projects ability to remain relevant.

It is important that the details developed from the process of MikroArkitekture inform people about a narrative, not necessarily of the past or present but also of the future. The details encourage and situate the architecture within the site's history, while beginning to engage with people to activate a once unknown or unseen narrative. In Nicholas Temple's work Disclosing Horizons: Architecture, perspective and redemptive space, he writes that the relationship to space, as human beings, is a reciprocal one with the assumption that architecture looks back at us. ${ }^{\text {ii }}$ The dialogue between a person and a space always informed by a presiding historical background which architecture gives it form. .ii $^{\text {Temple }}$ further suggests that humans are historical beings who are active participants in everyday experiences that create new spatial conditions that pay homage to past paradigms and anticipate future ones. ${ }^{\text {iv }}$ With regard to projects by Enric Miralles, Zabalbeascoa highlights that he defines the history of buildings not just as a 
SECTION 4.2

history of their own construction, but as a history of their sites. The history of a building can thus be seen as a beginning before its construction, and a new building should relate to the memory of the land. ${ }^{\mathrm{v}}$ The materials of the details emphasize a tactile quality both physically and visually while this play between form and texture produces an affect that stimulates the growth of the project to become not only a representation of the history and its narratives but rather to become an extension of it.

\footnotetext{
i Leatherbarrow, Uncommon Ground, 202.

ii Nicholas Temple, Disclosing Horizons, 267.

iii Nicholas Temple, Disclosing Horizons, 267.

iv Nicholas Temple, Disclosing Horizons, 267.

v Anatxu Zabalbeascoa, Igualada Cemetery, 10.
} 
SECTION 4.3

\section{DESIGN}
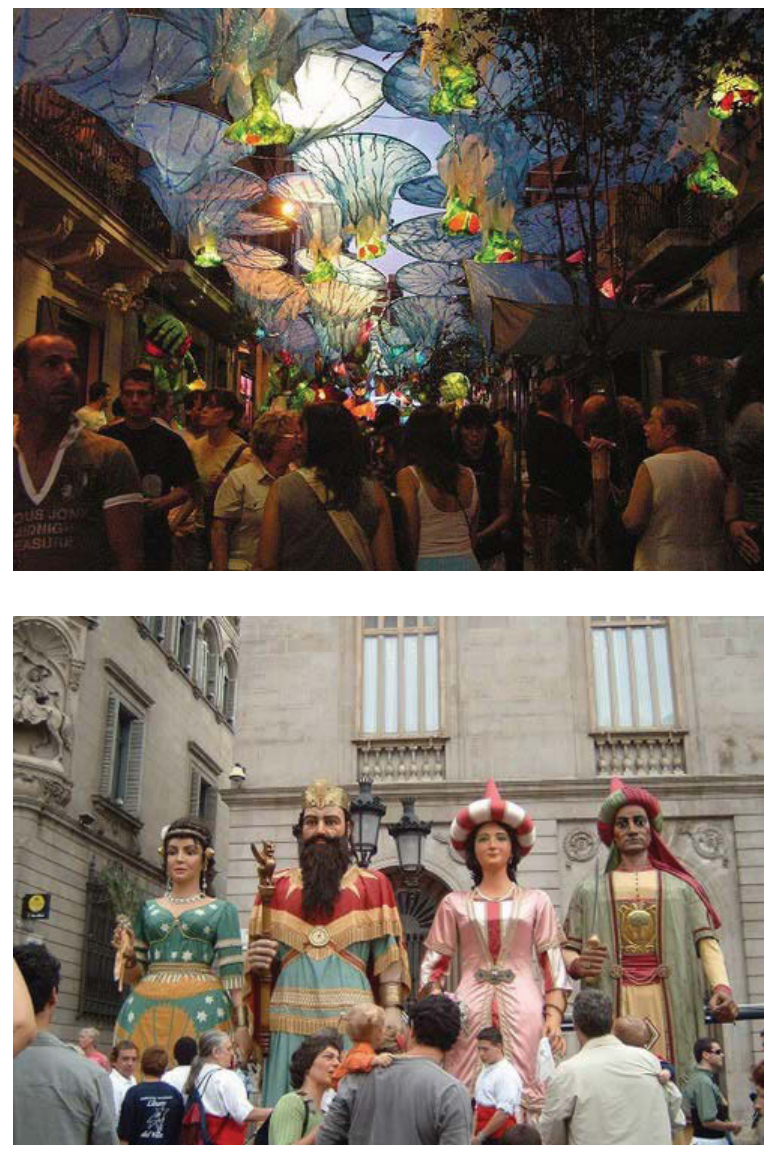

29. Festa Major de Gracia - canopy structures

30. Festa de La Merce - procession of Giants 
The principles of Mikro-Arkitekture can be applied to any scale of site but it is set to certain boundaries and conditions created by the site and its history. There must be a certain level of obtainable knowledge with regard to a selected site. Obtainable knowledge should be in some form of recorded documentation, either writings, photography, art, or film, which this information should be accessible to the architectural investigator. As one of the fundamental rule of Mikro-Arkitketure, the narratives must derive from a history and cannot be fictitious. Although present conditions of a site can be analyzed in order to understand the current historical topographical condition, this would only show a single layer, which is not sufficient in order to produce a proper sequencing of the narrative's associated details. The narratives must stem back to an origin point in order to maintain a continuity with the past, present and future conditions and narratives of a site.

With the case of Placa del Diamant, Gracia, Barcelona the site happens to have a very extensive history shaped by various festivals, events and conditions. When a site presents a profound depth of historical topographical layering this could make the historical analysis less strenuous but then their must be a more conscious awareness of which narratives to investigate and not to investigate. Although regardless of which narratives are used, they will always re-read the same history but each narrative produces a specific emotional response and this 
should be taken into consideration. The juxtaposition of various emotionally themed narratives add to the complexity of the topographical layering and can provide a wider range of details. The way in which these details would be developed should engage with events and ordinary life that occur in and around the site. This helps to anchor the project to not just the micro boundaries of the actual site but also in a more macro context of the site within the city. This transition from the macro to the micro helps to strengthen the connection between the topographical layers as they are re-organized and re-mapped as a new segment of the site historical interpretation.

Analyzing one of the main elements of the design, the canopies developed from the re-telling of various narratives acting on multiple historical topographical layers which each individual detail of the canopy responds to specific narrative. Firstly the canopy arms derive from the narrative of the Castellers, they are a group of Catalans who construct a human tower and occurs primarily during the Festa Major de Gracia but it also does occur during other moments of the year as well. Groups of people gather in a circular pattern interlocking arms creating a strong base for the construction of the human tower. Older and stronger participants are used as the base and as the tower builds the participants become younger, which the top of the tower if usually a 6 year old 

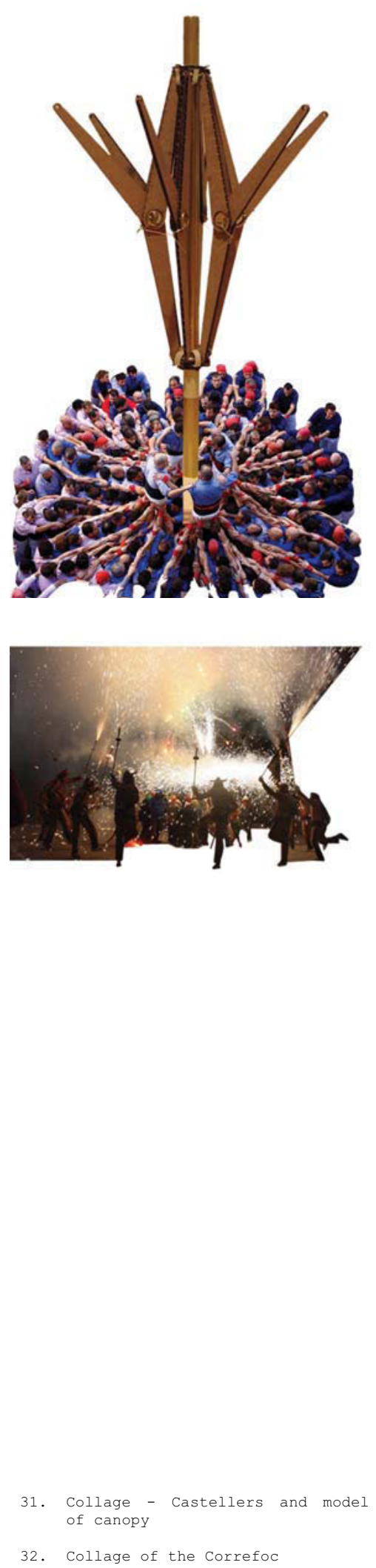

girl or boy. This narrative is juxtaposed with that of the Correfoc, this is an event that is part of La Festa de la Merce. This is a festival with medieval origins which a group of fire demons dance and parade through the narrow streets in order to ward off any bad spirits that plague the city. This symbolizes a medieval tale when the city was plagued with a swarm of locusts that had destroyed many farm crops and the citizens began to starve. The Bishop and people prayed to the Virgin de la Merce to relieve them and once their prayers were answered they commemorated her kindness with this annual festival which has evolved into the modern day celebration of fire demons and giants (shown in figures ?? and ??). Thirdly, the overall idea of the canopy stem from another event that occurs during the 
SECTION 4.3

Festa Major de Gracia, nets are strung between buildings to create a roof-like structure (shown in figure 29). From this, people hang various shaped lanterns and decorations that cover streets around the plaza. While the narrative of the canopy portrays a singular object its details speak to a variety of influences which are developed from the overlapping and re-sequencing of the historical topographical layers that are rooted in the sites' history.

This ability of Mikro-Arkitekture to respond to these human condition is what activates and assures its placement within a site. David Leatherbarrow touches on this theme in 'Architecture Oriented Otherwise,' saying that the building's 'eccentricity' is its existence outside itself, for its behavior testifies to a constitutional weakness at its center, a negativity at its heart, because it must wait for its circumstances to give it what it lacks light, air, human events. ${ }^{i}$ The height of the canopies and the area that they cover is in direct proportion with each other. The maximum span is also the maximum height of the core component of the structure. This core itself remains static while the animated portion of the canopy - the extended arm-like structure changes depending on the user and the rituals taking place at a specific moment. A wench device placed inside the main core of the structure allows the arms of the canopy to be raised and lowered. This interaction that occurs between the users and the architecture allows the site to adapt to these new hybrid narratives while participating in old ones as well which 
SECTION 4.3

each detail is essential in completing the re-telling of a history. In the writings of Leatherbarrow this interaction between humans and architecture is a performance which architecture's performative labour has no end as it must continually present itself anew with each individual performance or interaction. ${ }^{\text {ii }}$

Another defining narrative brought back in this reading of the site's history through the design process are the systematic air-raids that took place during World War II by "the Franquist, some rogue squadrons, others by Marjocan-based planes, but most by the German and Italian airforce." ${ }^{\text {iii }}$ General laborers throughout the city gathered together to create what is known as the refugi antiaeri. They developed these intricate labyrinth system that went approximately 12 meters underground, weaving and intertwining to other refugi throughout the city. The steep stairways and curving corridors were created using the volta catalana technique this allowed for the passages to be wider and longer, providing more people to seek refuge. Various entrances were planned so that the people could expediently enter and to provide other means of exiting if one or more entrances had caved in during an air raid attack.

A British Scientist by the name of John Haldane researched the refugi antieri in order to incorporate a similar system in the UK, which the country was devastated by similar bombing raids. In his report he describes the construction of the tunnels: 
"In this area there were four entrances which led down by ramps with a few steps, to the tunnels. The ramps twisted repeatedly, until a level of about 55 feet below ground was reached. Here began a labyrinth of passages about 7 feet high and 4 feet broad. They were cut in the very tough soil of the district, and had no lining, and I think no supports such as pit-props. They were, however, being lined with tiles with a cement backing so as to give a semicircular arch, and vertical walls. Lighting was to be electric, but none was yet installed. Ventilation was to be by electrically driven pumps, and a vertical shaft which had been use during the excavation was to continue as a ventilating shaft." ${ }^{\text {iv }}$

Once enemy airplanes were spotted from the lookout towers located near the waterfront of the city, residents had one to two minutes to make it to one of the nearest refugi. This network of underground Catalan vaults extending several kilometers, is a modern development in the long history of the Catalonian capital. Montalban writes that "[at] the end of 1938, Barcelona was a city of survivors rushing in and out of the metro or the network of shelters which had been built, some of which survived for years as a memory of collective terror."

There is an attempt in the project to raise peoples' attention about the tunnel system up from the ground and have it presented in the plaza as a physical historical moment. The concrete formworks are extrusions of the stairways used to access the underground tunnels. This particular section of the topographical layer is thus extruded as it passes through the other strata and comes into its final form. The purpose of these components are intended to function as a type of sitting area. This idea of sitting in contrast to the quick movement of running into 
SECTION 4.3

the tunnels offers a point where reflection between the past and present can interact.

There are strips of reveals that penetrate the plazas slate stone finish and continue downwards into the tunnels offering pedestrians a quick glimpse into this narrative. They derive from this idea of the main ventilation shaft which would allow air, light and sound into the tunnels. The reveals allow light to penetrate into the tunnels and vice versa, lights could be planned inside the tunnels in order to articulate specific features illuminating moments of the narrative from below. This literal act of opening up the tunnels breathes life into the underground narrative. When entering the plaza and standing above the reveals one can see a specific portion of the steps below through the slightly tinted tempered glass covering. The reveals art meant to capture this act of linear descent which one end is narrower than the other, this visually speaks to the user and allows them to participant in the act. Standing from the narrow end and looking to the wider portion emphasizes the act of emerging from the tunnels, this would be the opposite affect if the user was to stand at the other end. The concrete formwork derives from the translation of certain highlighted sections of the tunnels which they are meant to emphasize this important moment of descending. They capture the curvature of the winding tunnel and mimic this expression of the Volta Catalana. The formwork plays with different heights in 
SECTION 4.3

order to allude to the change in depth as on a person would make their way down the steep winding steps, reaching a landing before descending even further. This detail creates a direct visual interaction between this topographical layer and the visitors. A dialogue takes place developing an emotional response through the mikro-detail; these reveals into the ground enhance the re-telling of the narrative by acting as a mask, highlighting but does not fully revealing the tunnels. In Antoine Picon's writings on Ornament, he states that [masks] conceal and reveal at the same time, in addition to disguise and transform, concluding that revealing identity is another reason for masking. ${ }^{\mathrm{vi}}$

The individual details do not distort the historical topographical references but rather they provide a source of bridging the connection between the narratives and what occur above and/or beneath the plaza. The vertical translation and movement of a detail creates points of intersection with the other layers. From this, the details begin to interact with each other as they re-tell a new story. This vertical shifting through the strata creates projections that frame these moments of interaction that occurs between the layers developing a historical collage that elaborates and introduce the new elements into the project which was not visually present. From this collage, objects can be discovered and become tangible components representative of the architecture. This movement between strata reinforces this notion of macro to microanalysis. This assists in 
creating a direct correlation between what is being revealed and the design while strengthening the connection between the three main components; the history, the narrative and the detail. The action of photomontage is strongly used throughout the work of Enric Miralles, where he states during a discussion that:

"The assembling of the photomontages brings the construction back into the studio, making it the place of transformation. The various projects being to appear as modifications and reiterations of each other. Each has a different starting point, each grows towards its specific requirements. But all are born in the same place, all are woven to respond to topological conditions."vii

This helps to emphasize the intermingling of various narratives and regardless of its' time, as long as they come from a similar history, its re-organization will always lead back to the same point of origin. It is important that the overall history provide a common means of recognizing the narratives in order bring the details together and that this connection exists throughout each layer. This allows for a cohesive representation of all elements involved in the design making it possible to further the development of a project through the process of MikroArkitekture. 
SECTION 4.3

i David Leatherbarrow, "Architecture Oriented Otherwise," (New York: Princeton Architectural Press, 2009) 62.

ii Leatherbarrow, Architecture Oriented Otherwise, 62-63.

iii Manuel Vazquez Montalban, Barcelona (London, UK: Verso, 1992) 130.

iv John Burdon Sanderson Haldane, A.R.P. (London, UK: Left Book Club, 1938) 24.

v Montalban, Barcelona, 133.

${ }^{\mathrm{vi}}$ Antoine Picon, Ornament: the politics of architecture and subjectivity (Chichester: John Wiley and Sons, Ltd) 61.

vii Miralles, Mixed Talks, 39. 


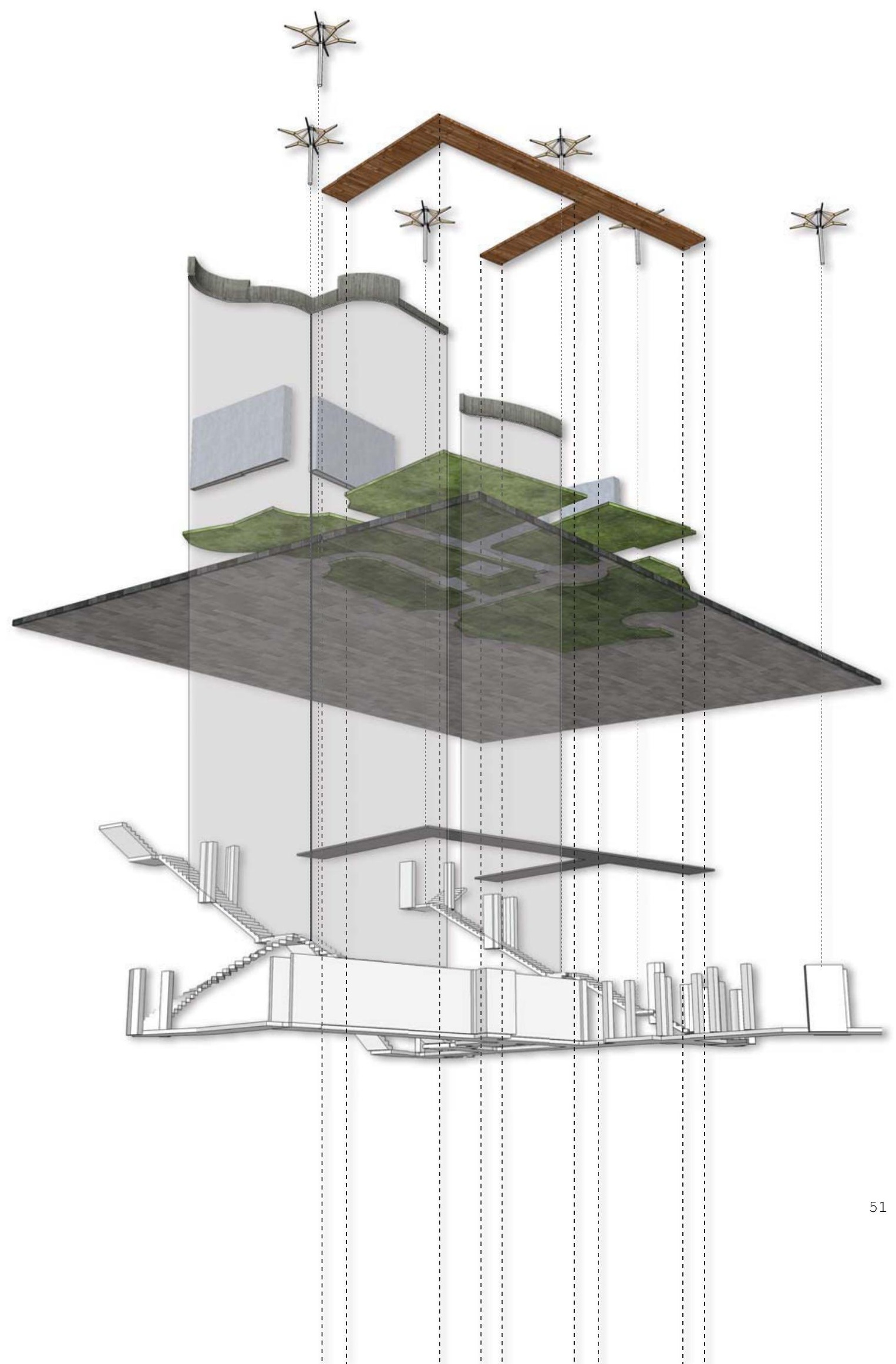



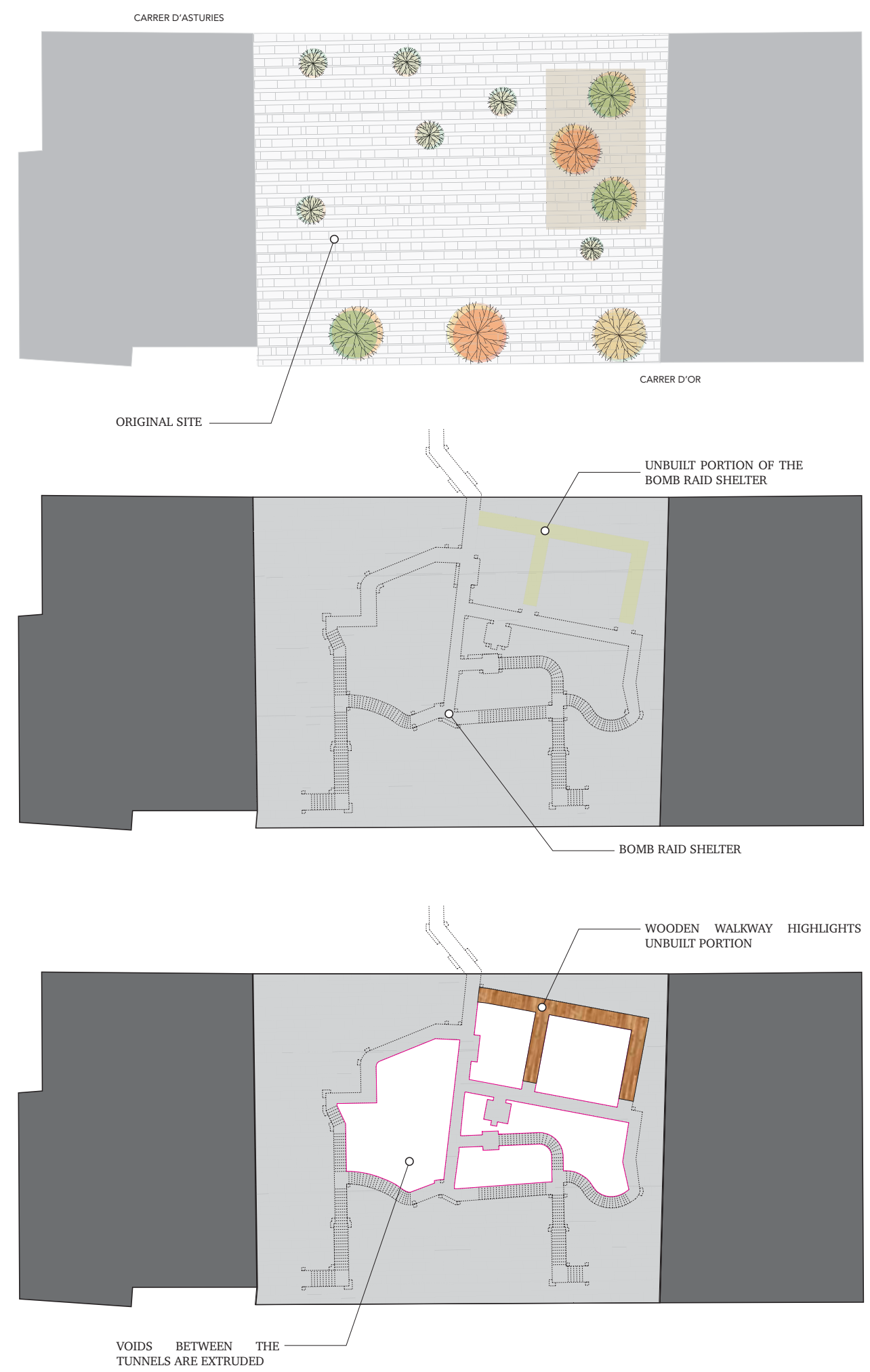


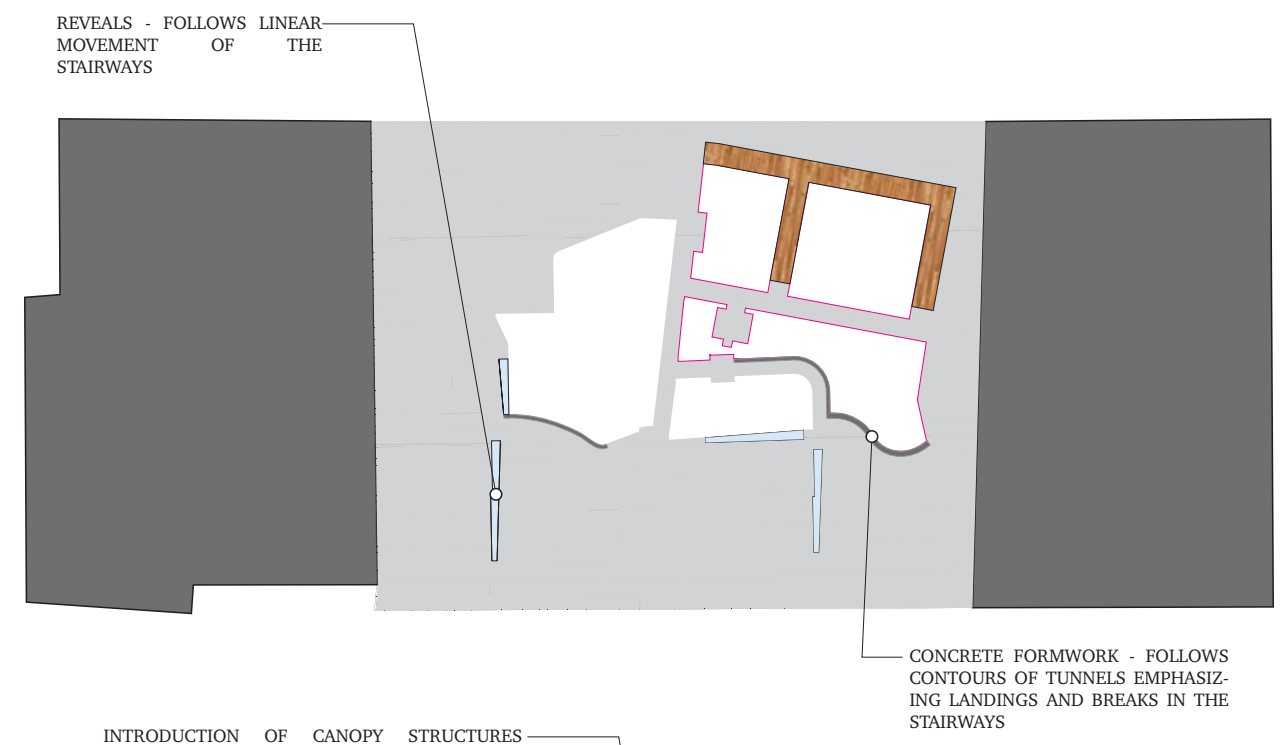

INTRODUCTION OF CANOPY STRUCTURES ADDING THE NARRATIVES OF THE CASTELLERS, CORREFOC AND THE FESTA DE GRACIA
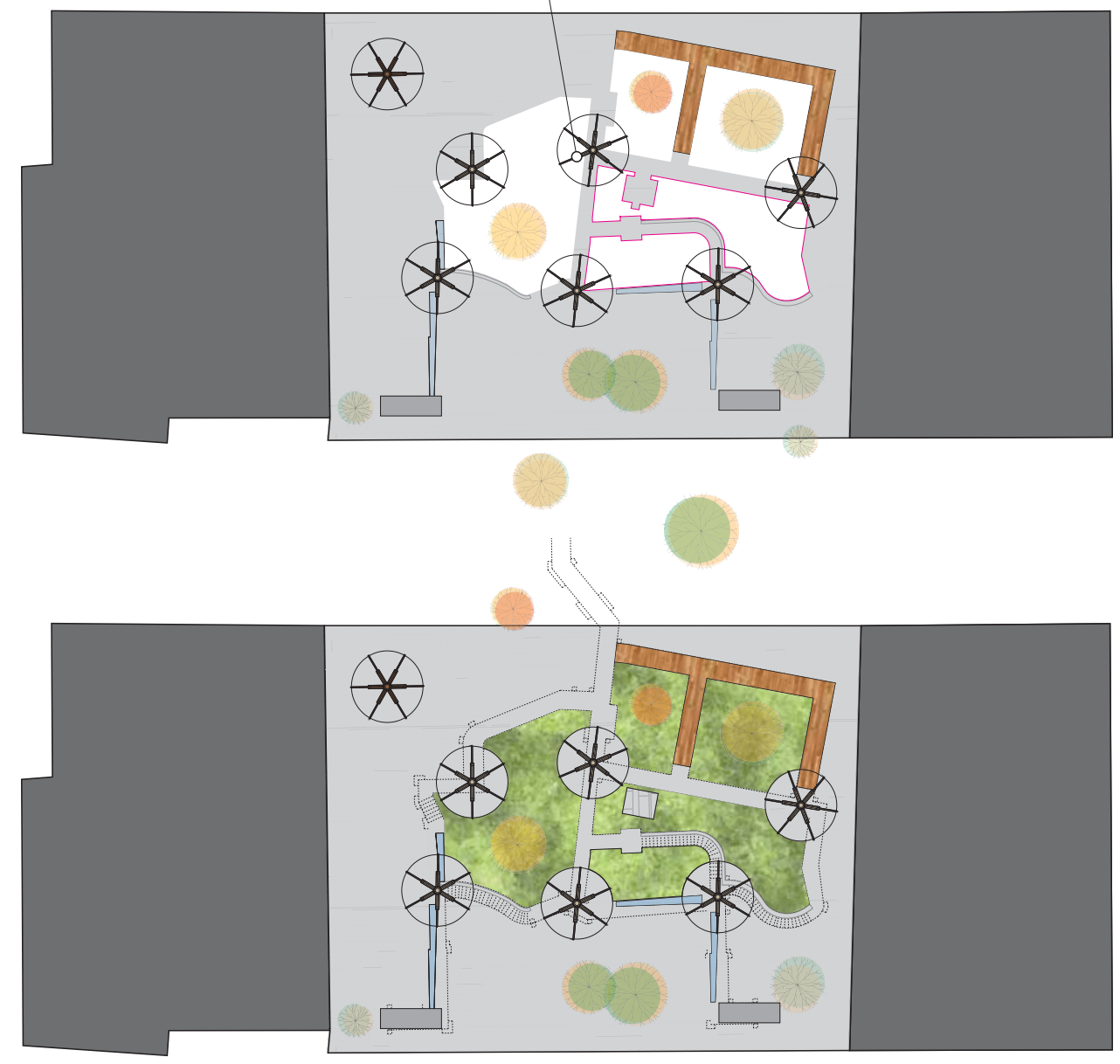
SECTION B

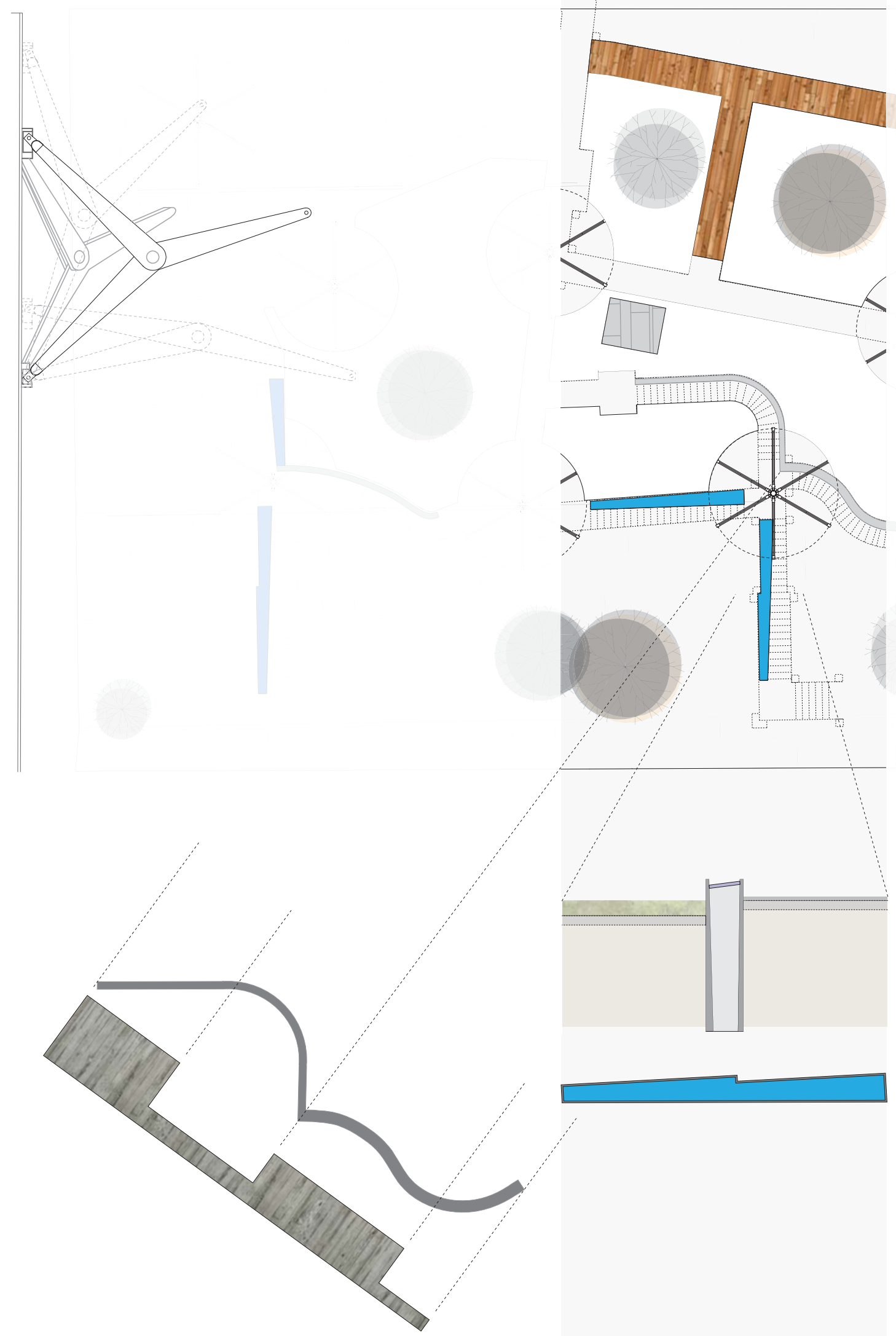





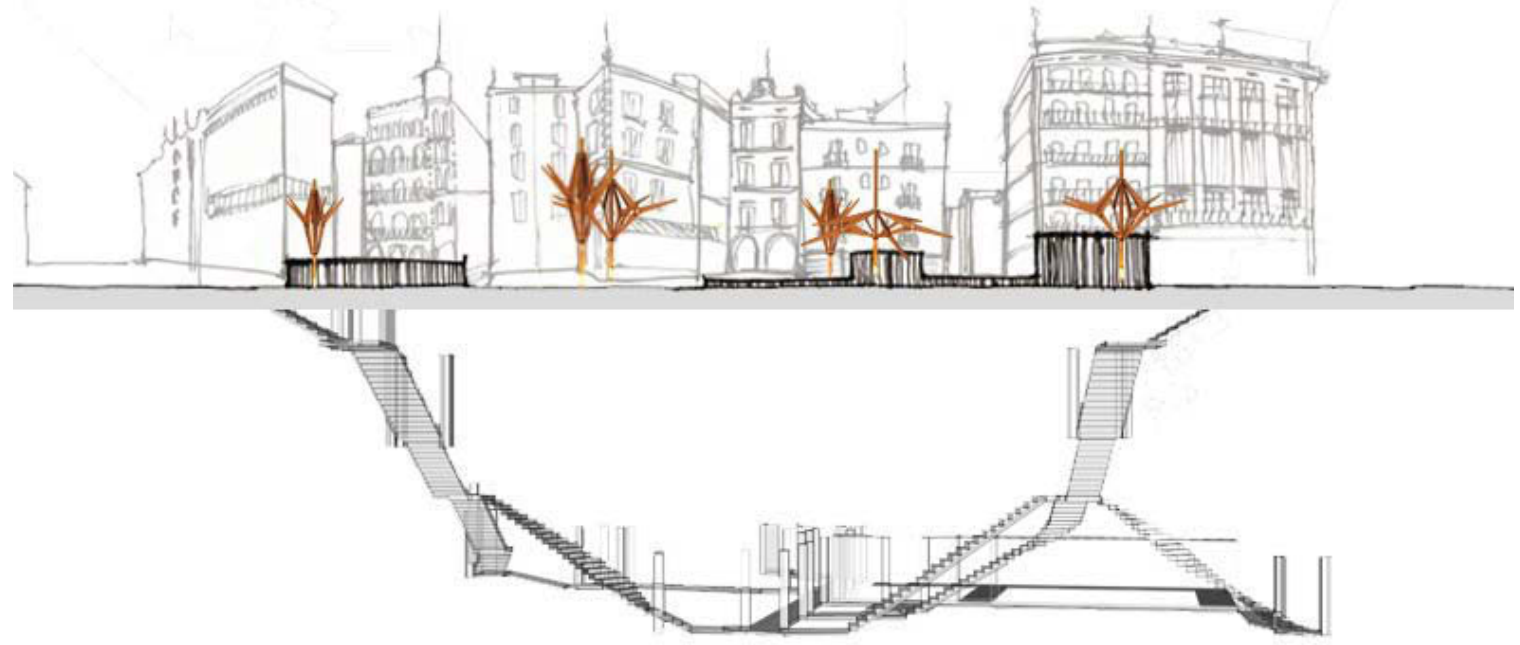




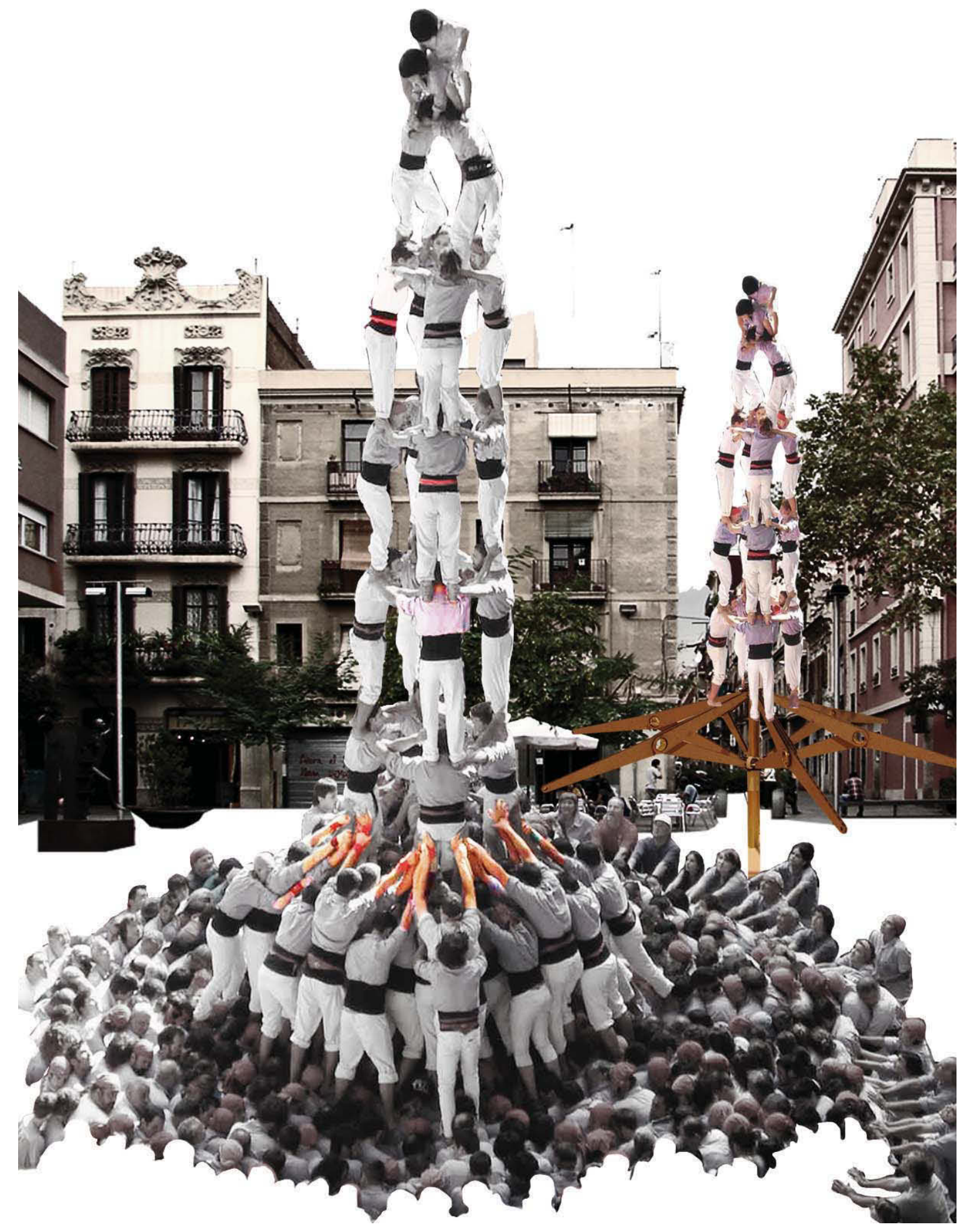



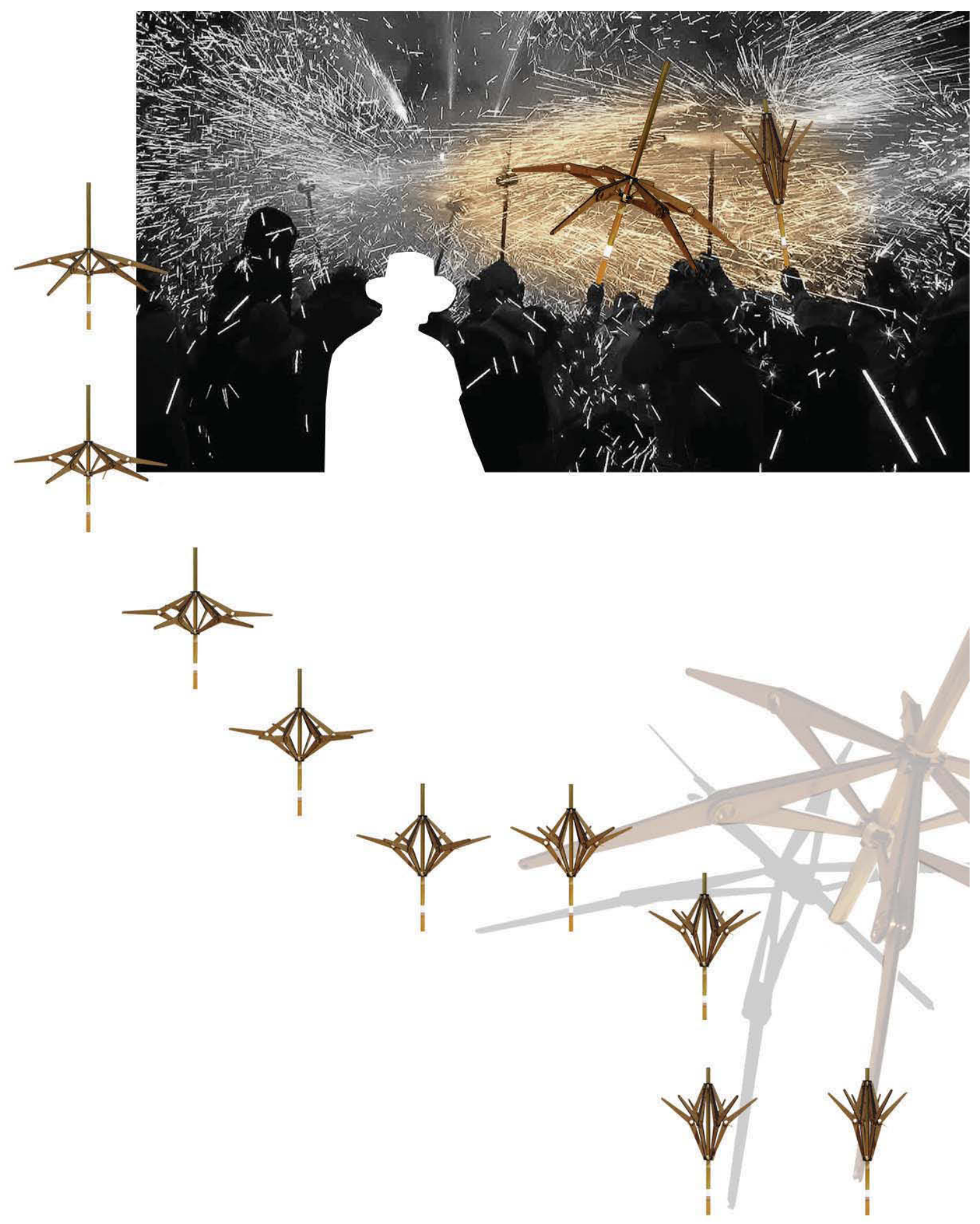
SECTION B
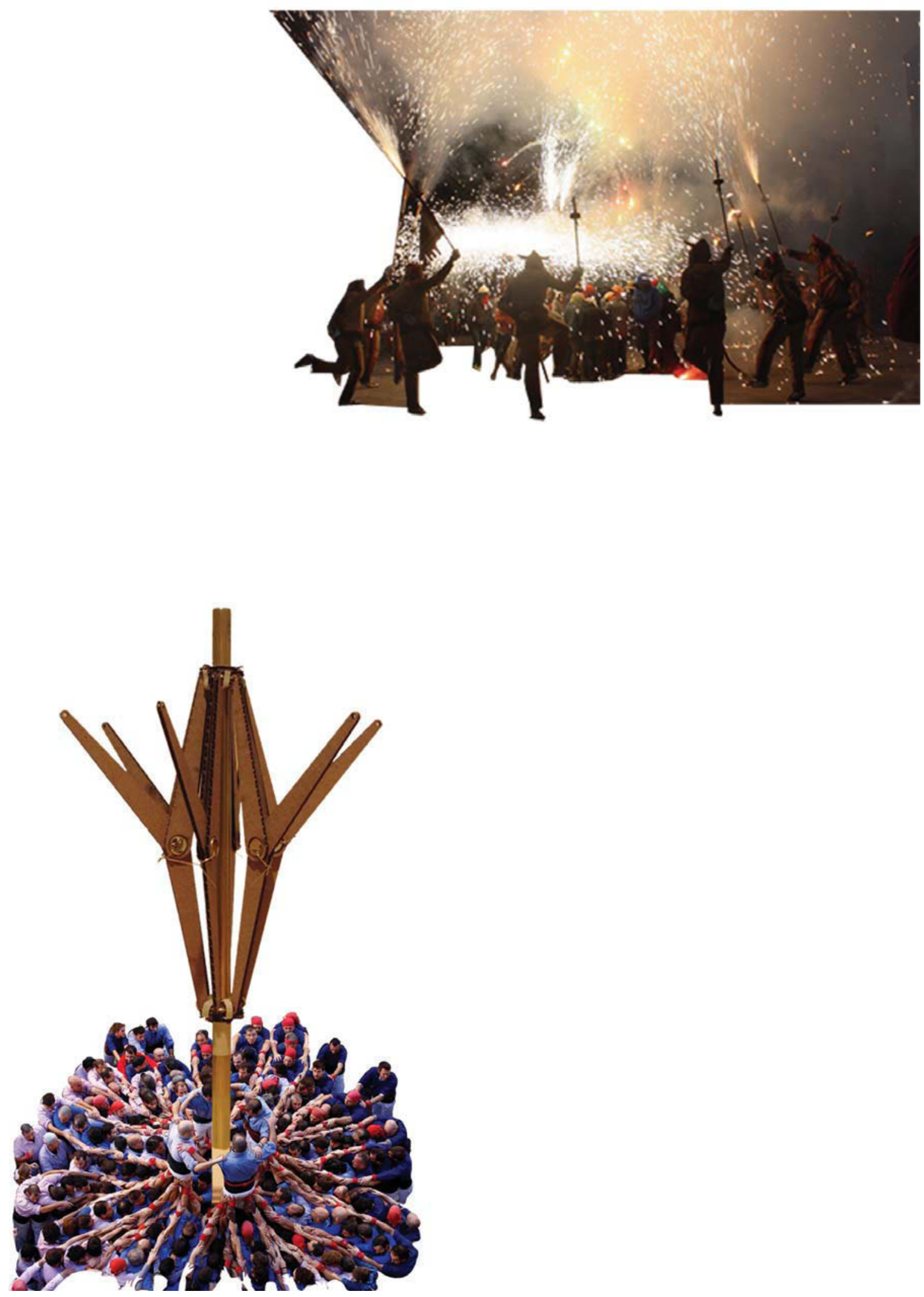


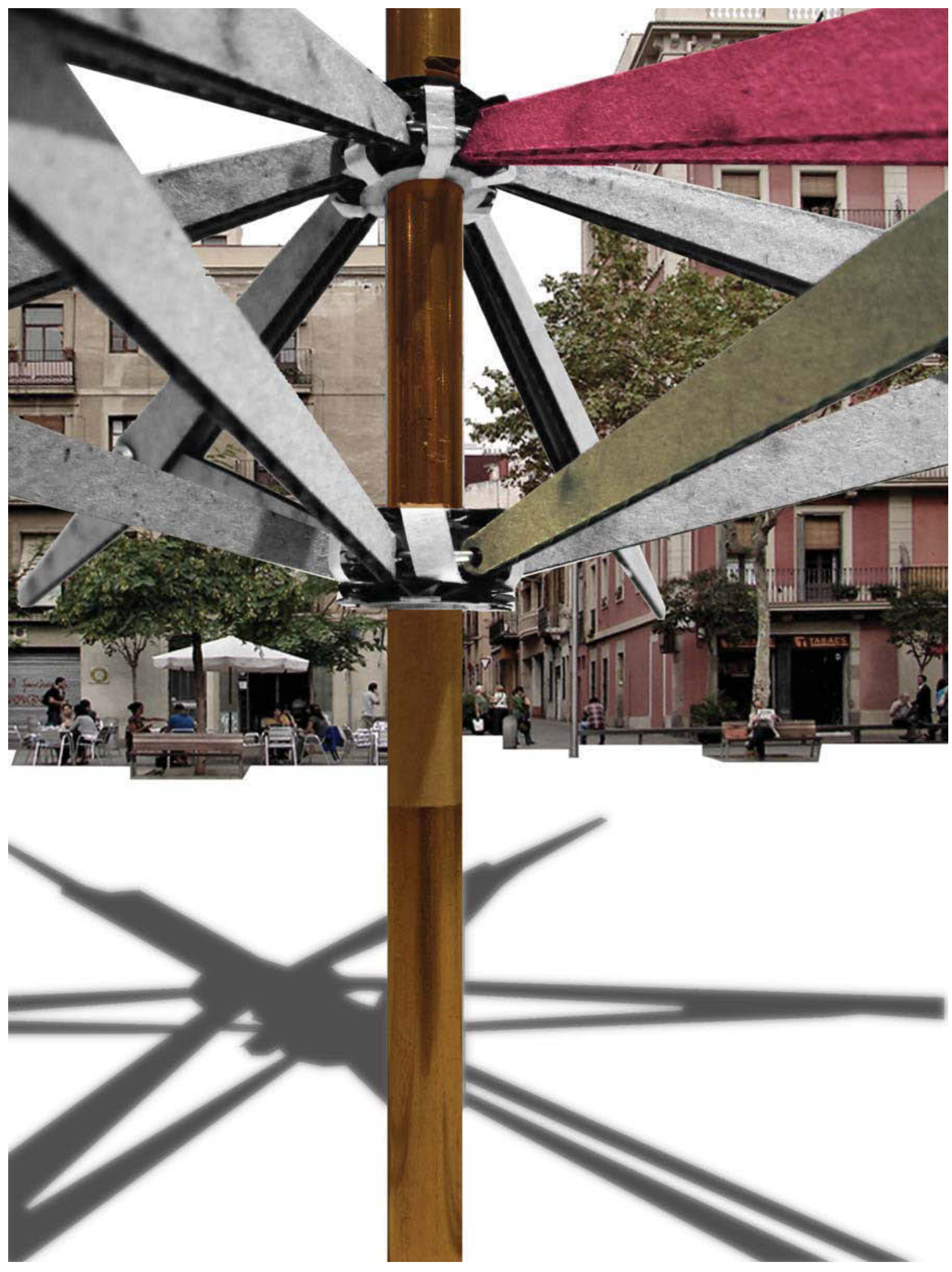


SECTION B

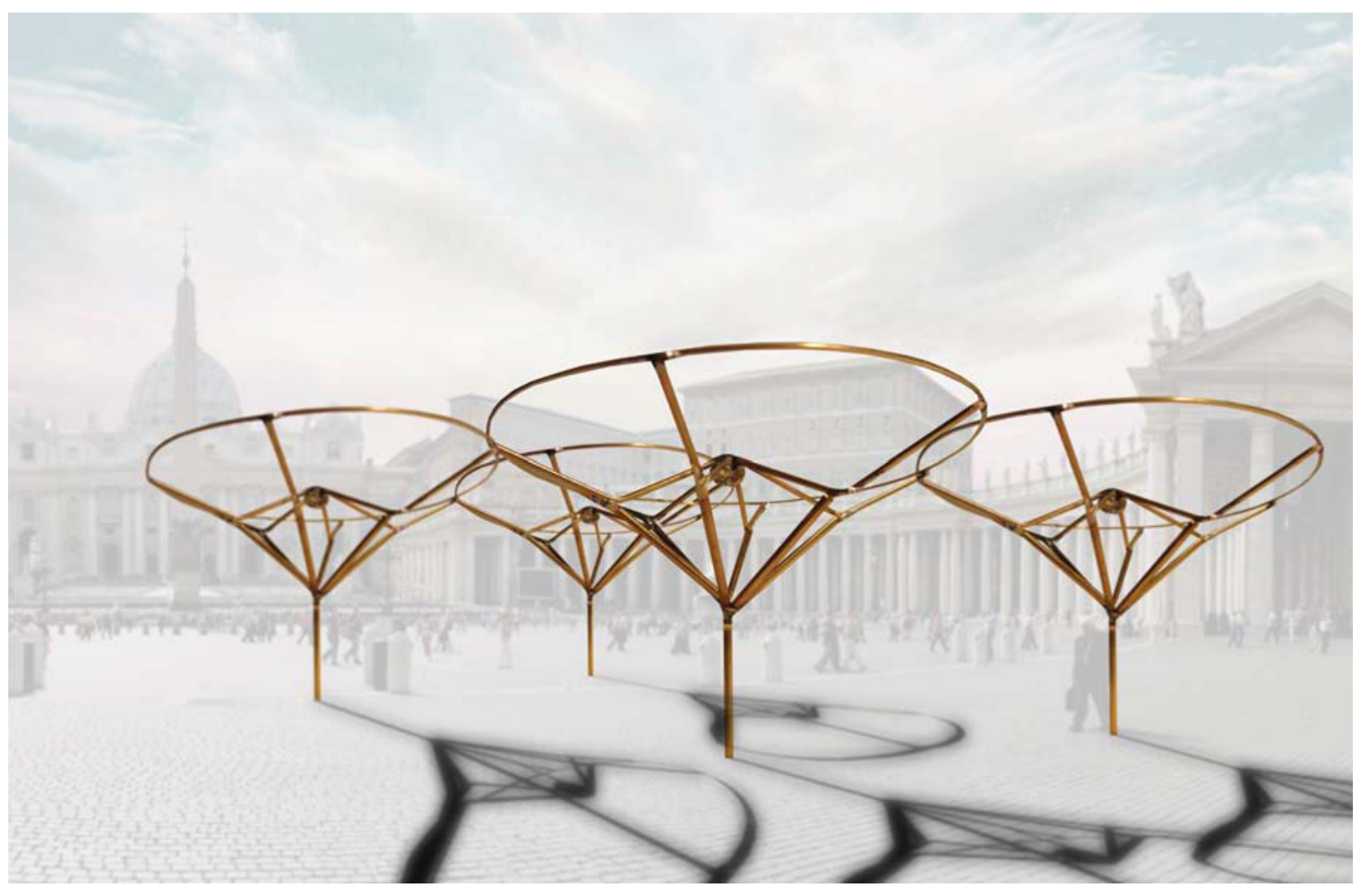




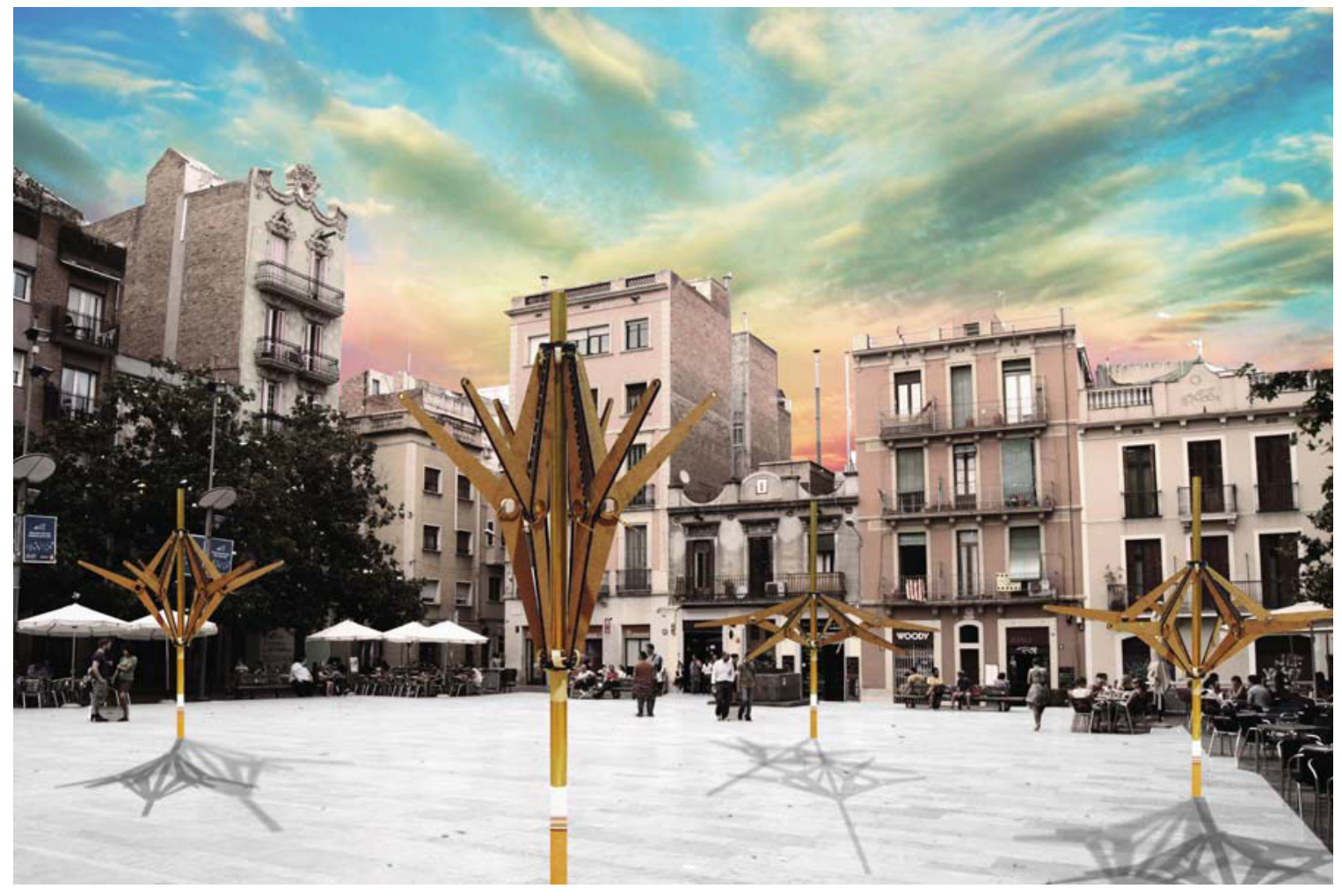




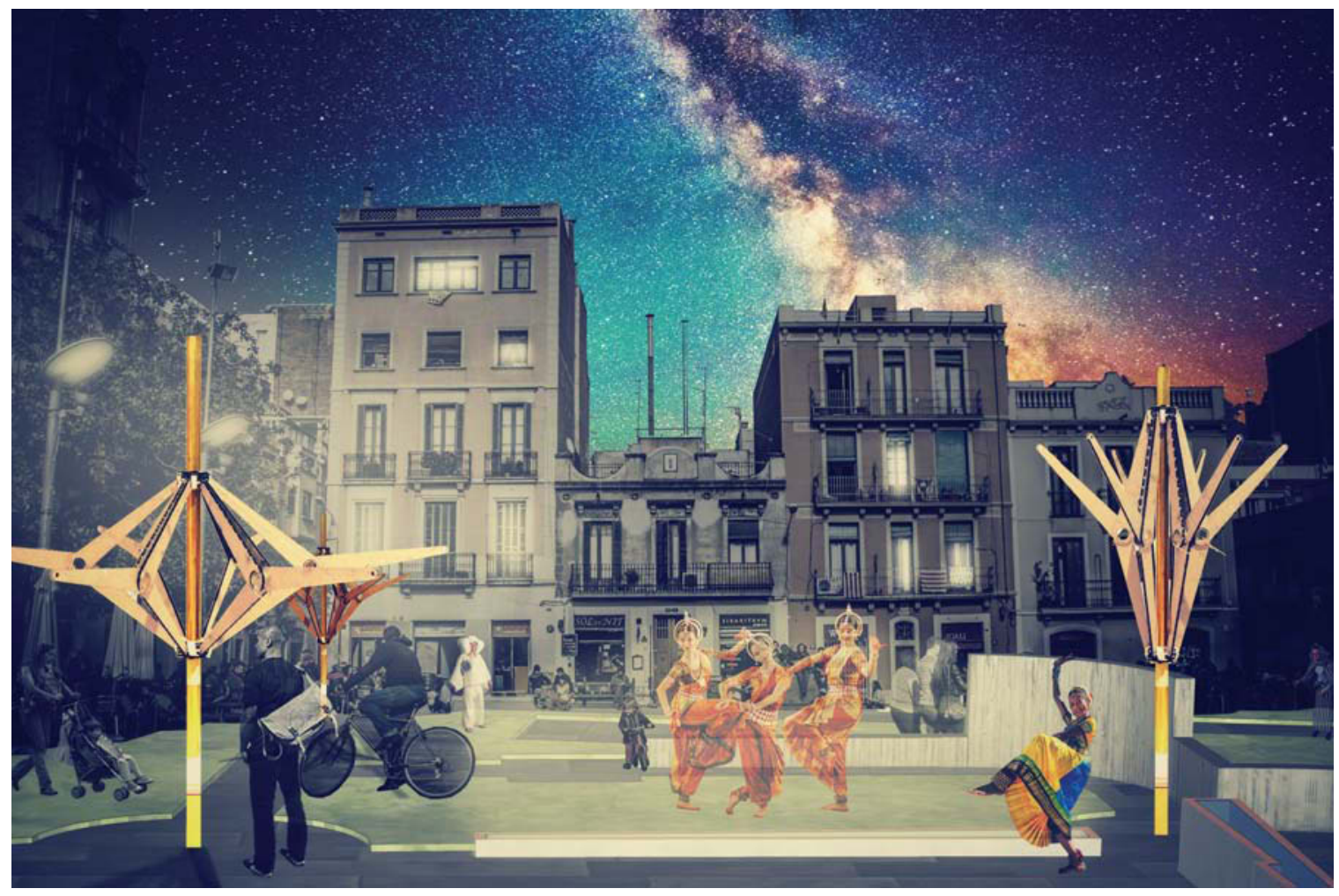


SECTION B

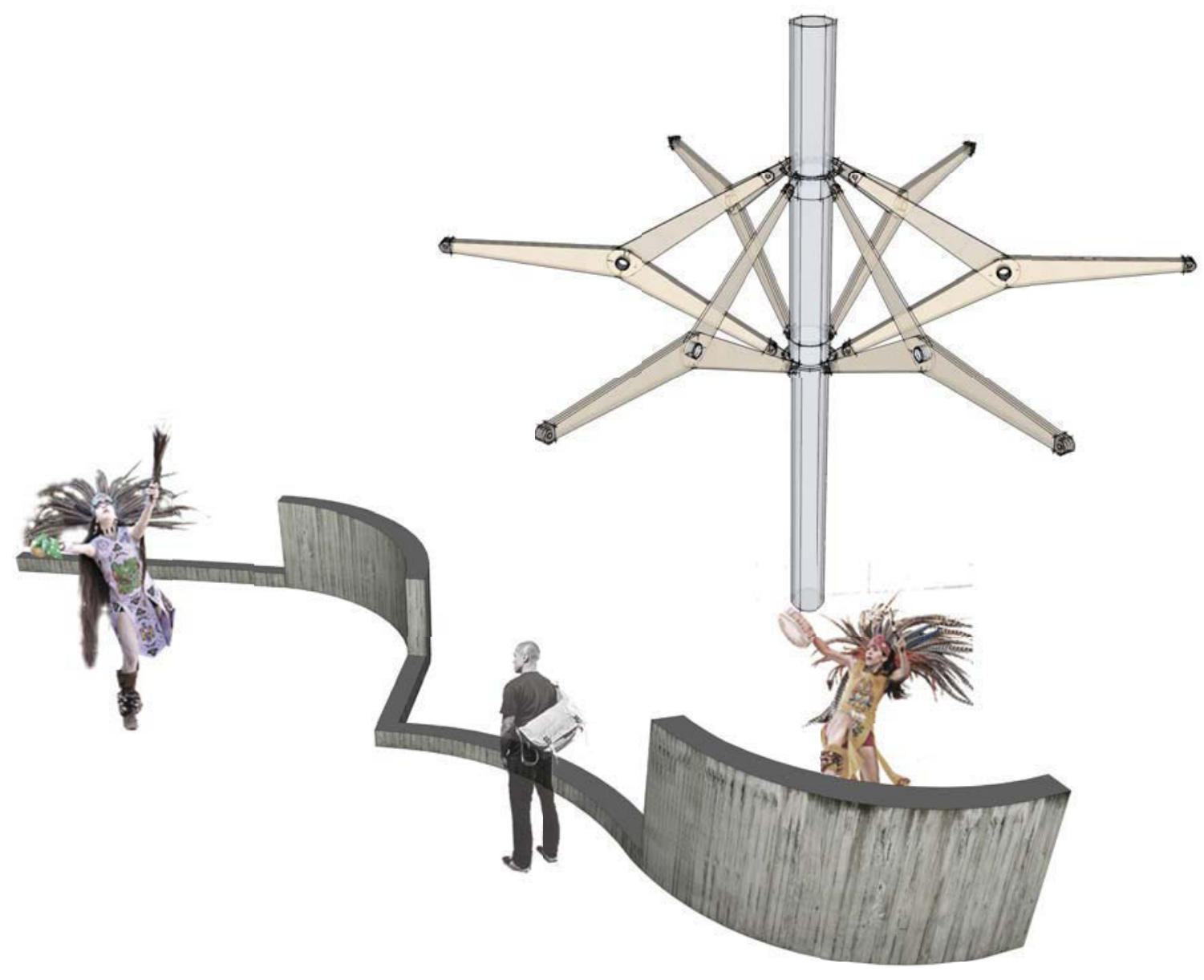


SECTION B

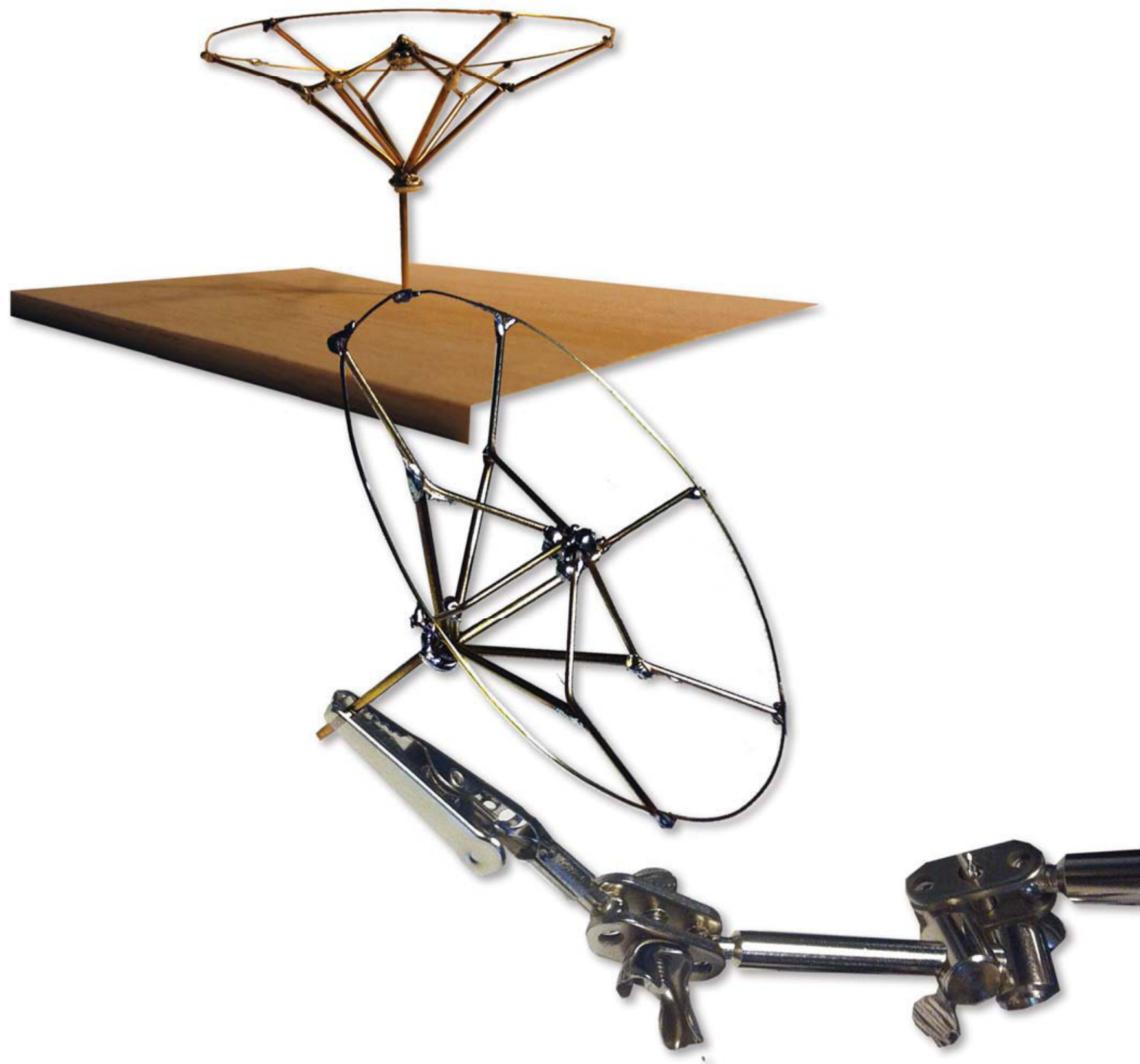


SECTION B

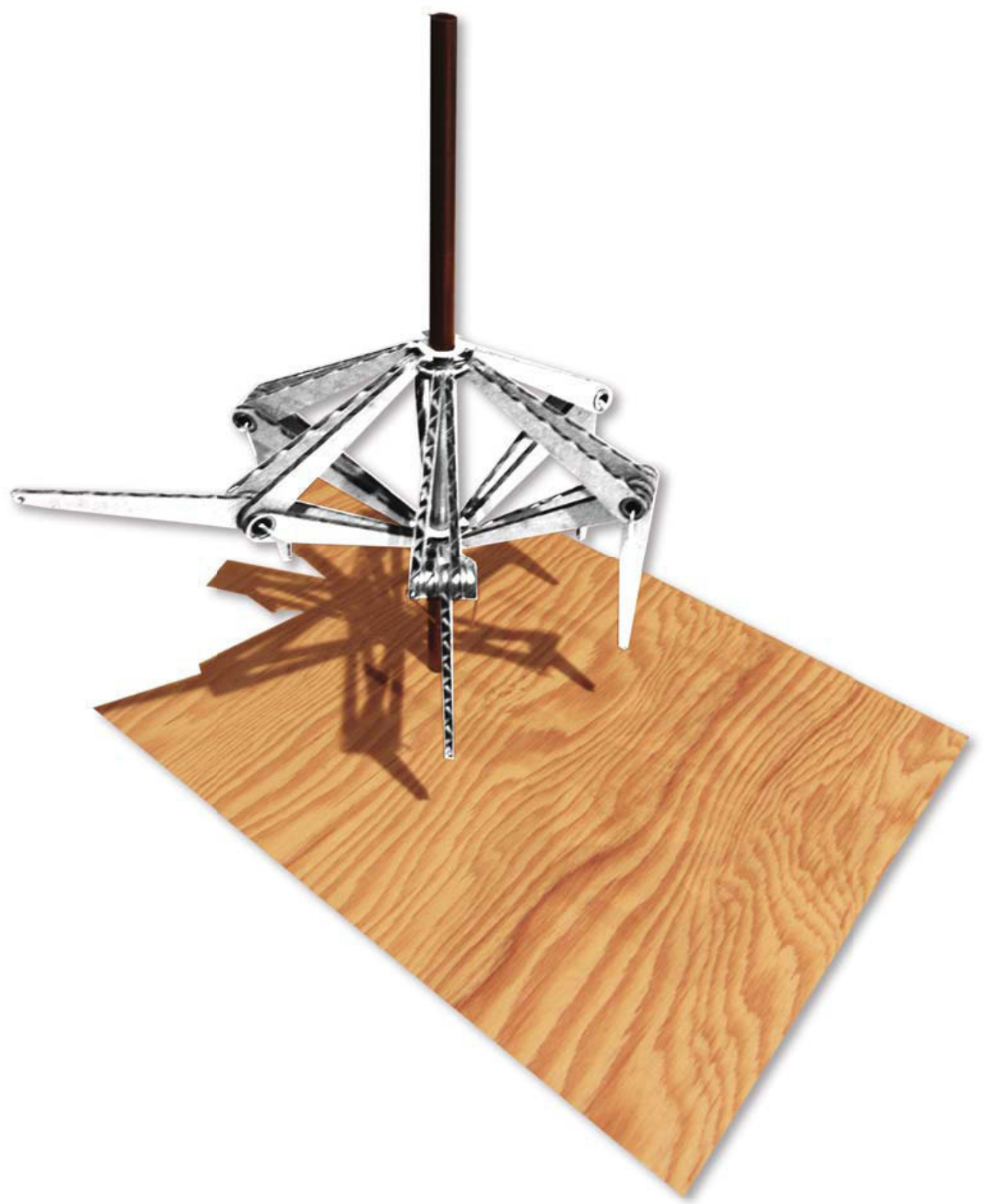



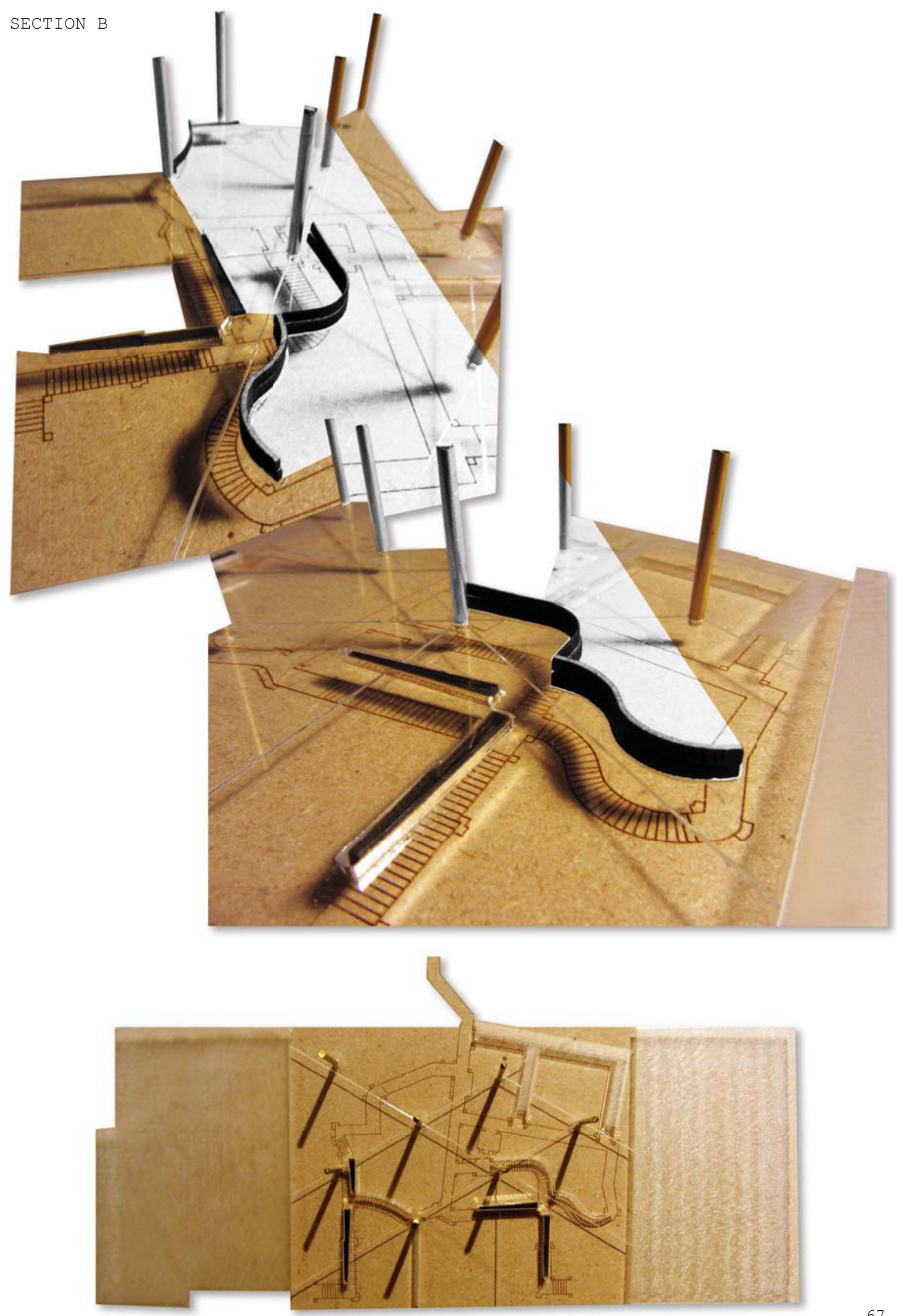


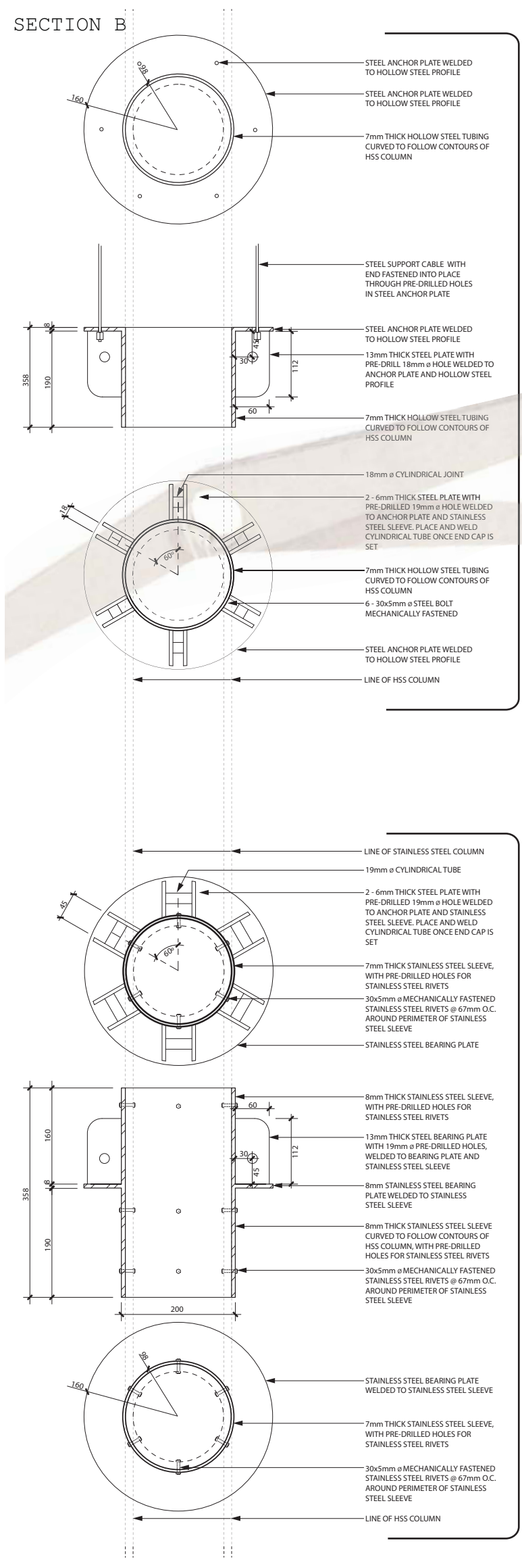


SECTION B
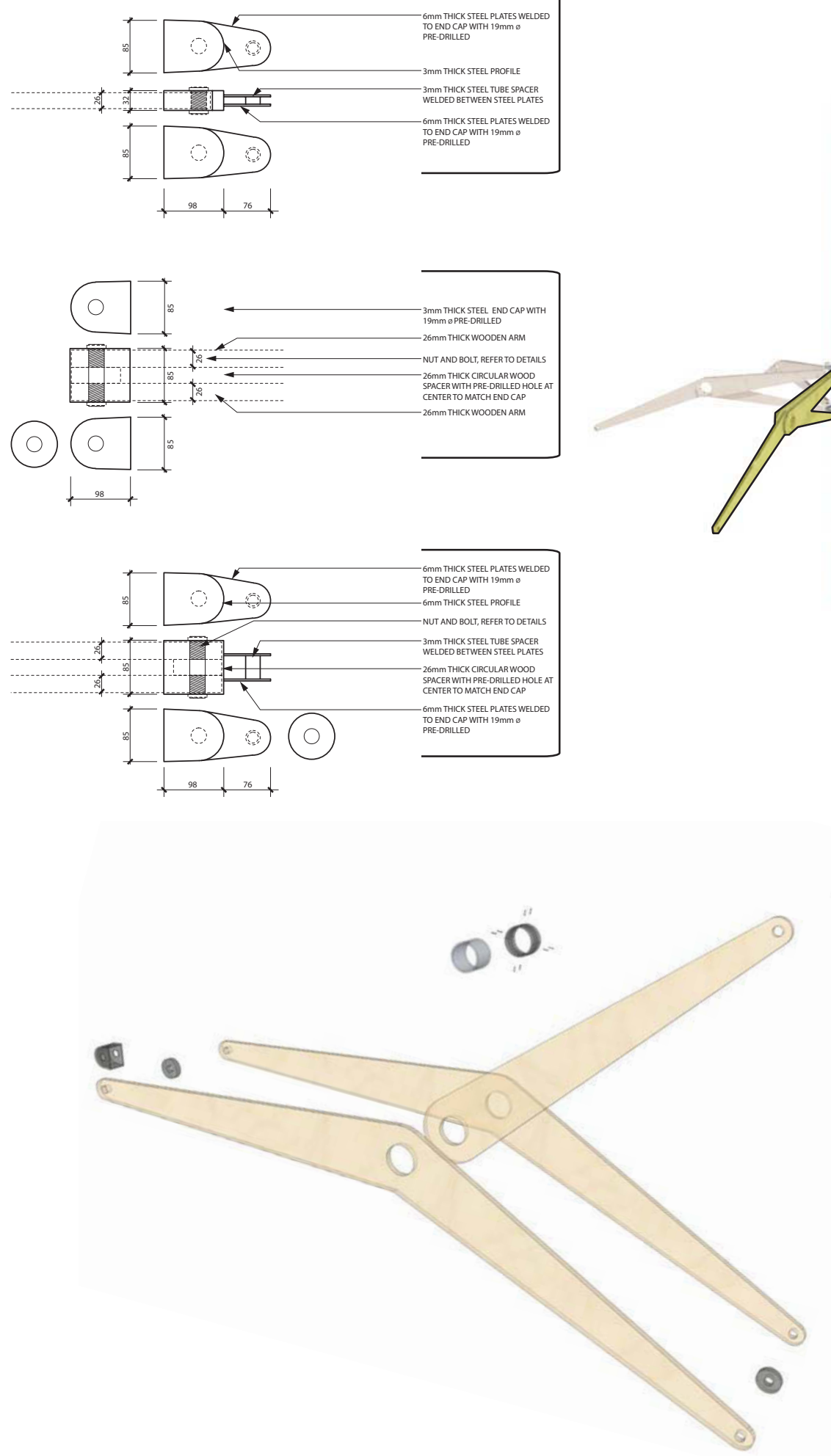

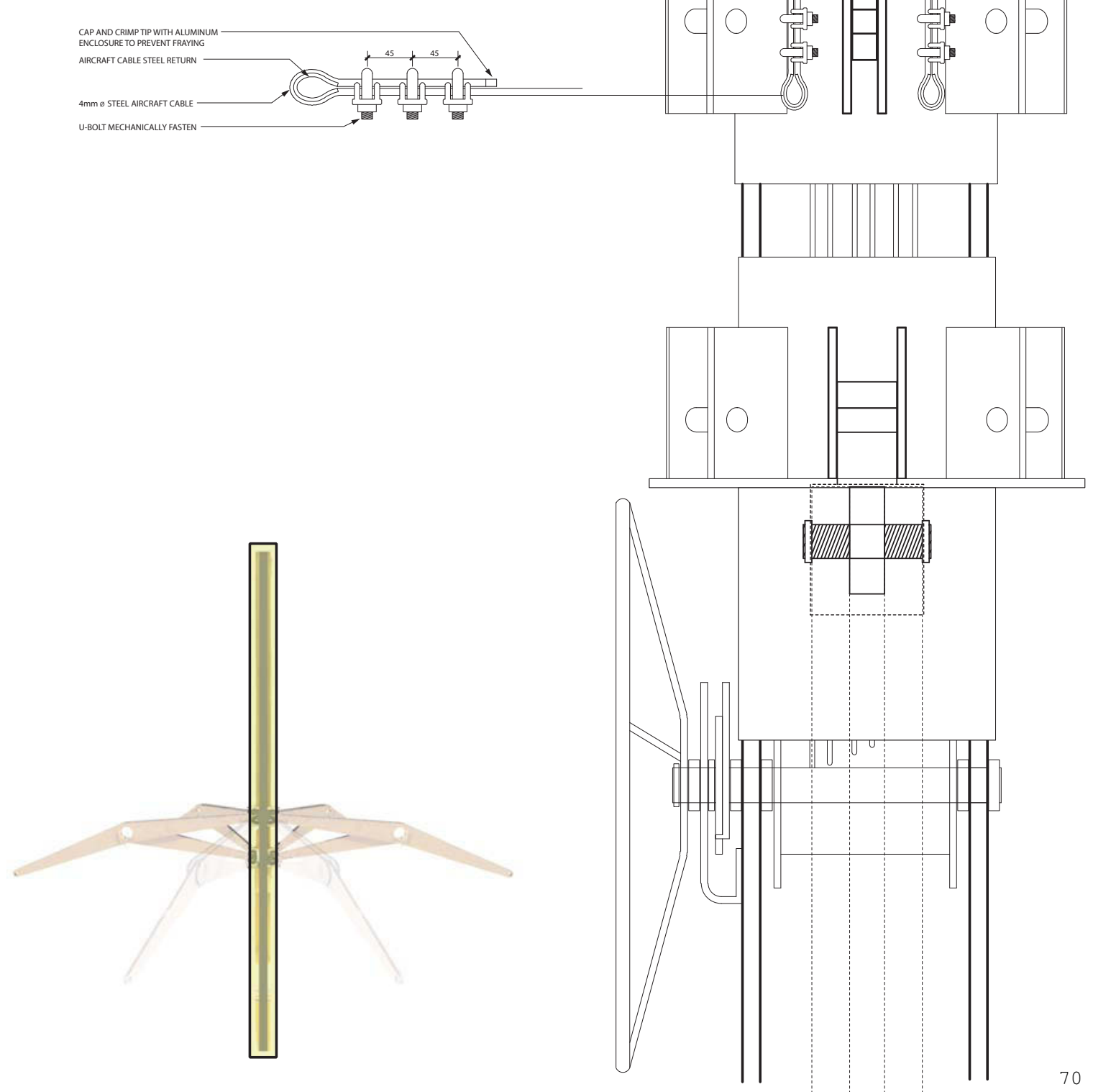


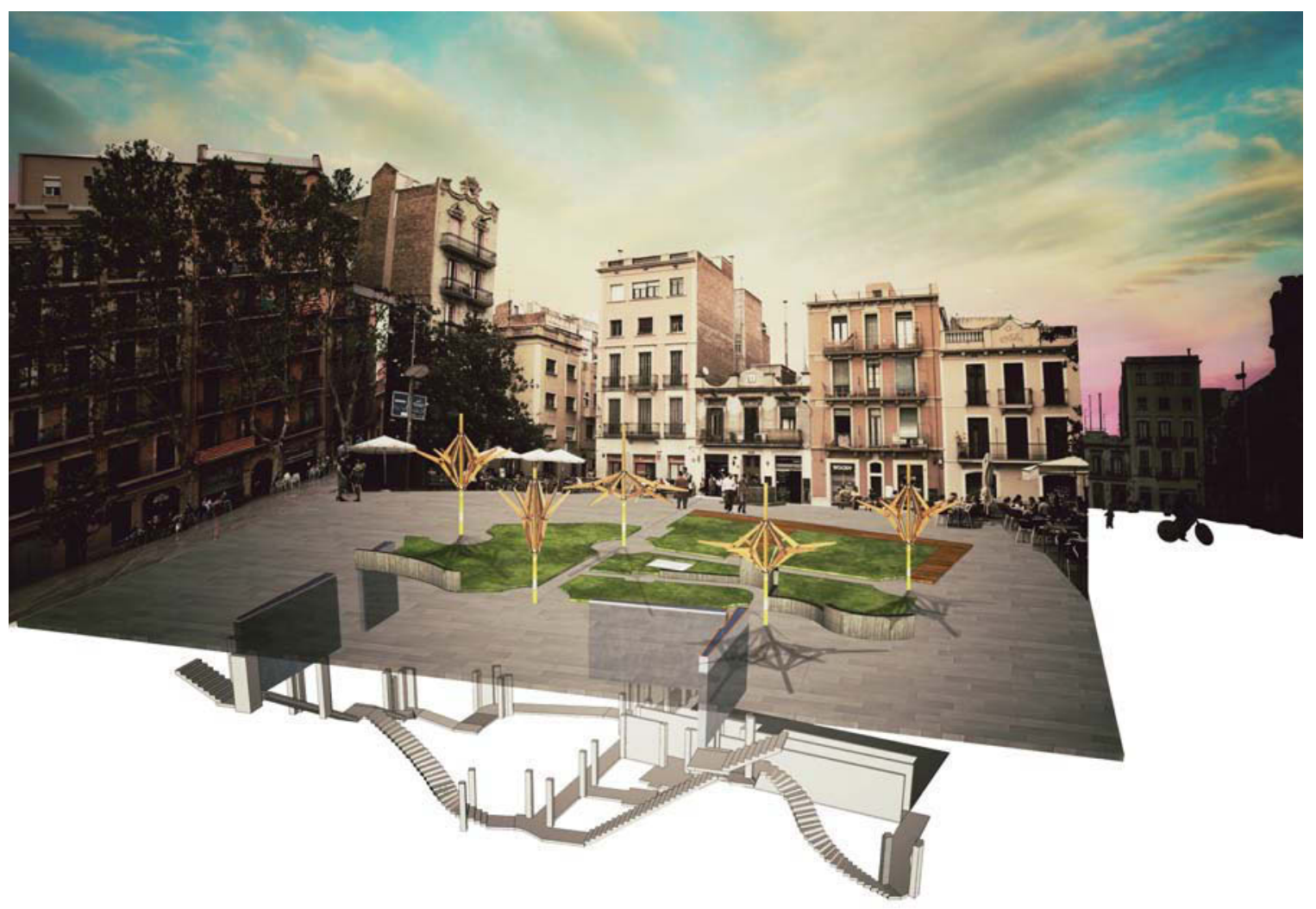


figure 1:

The image illustrates the architectural development through the analysis of the historical topography. It highlights the narrative of the bomb-raid shelter (refugee anteaeri) and how through this process of re-organization of the narratives forms can be extruded in order to bring particular details to the surface. This allows unknown elements to become visible and engage with other narratives.

figure 2-3:

The series of diagrams illustrate a plan view of the transformation and the translation of the information when shifting and re-organizing the historical topographical layers. They emphasize changes to the landscape of the plaza in subtle ways that interact harmoniously with the other forms/narratives. A natural growth of the details is shown in the transformation of the plaza. Details are developed in subtle ways introduce different textures and forms that create a tactile and emotional connection with a person. The final design of the site plan introduces green areas, glass reveals that penetrate into the bomb-raid shelter, concrete formworks, canopies and a wooden platform.

figure 4:

Contextual site plan that illustrates the different details that are formed through the process of Mikro-Arkitekture. It depicts key details such as the canopies, concrete formworks and tempered glass reveals embedded into the plaza. The reveals help to create a direct visual link between what is happening below the plaza and bringing awareness to an unseen narrative. They specifically highlight details of the stairways in order to give a sense of depth and movement while the formworks reflect a curves of the tunnels.

figure 5:

Collage of different narratives that are used in re-designing the plaza.

figure 6:

Conceptual section which helps to visualize the connections that are being developed from the juxtaposition of various narratives and how their interaction informs historical topographical development of a site.

figure 7-10:

Conceptual collage that illustrates how the interaction between the narratives can inform the architectural development of the project. It also demonstrates the idea of scale and tactility which are key components in the process of MikroArkitekture. 
figure 11:

Initial conceptual design for the canopy detail.

figure 12:

Final conceptual design for the canopy detail.

figure 13:

Final conceptual design for the concrete formwork and canopy

figure 14-16:

Final models of the canopy detail and site plan model which highlight the main features of the design (formworks, glass reveals and the canopies)

figure 17-19:

Micro-details of the canopy design. This micro analysis of the details allows for the development of specific components to be created that work together in order to develop a unique architecture that responds to the historical topography of that site and the associated narratives. The details encourage an interaction to develop between the user, the history and the architecture.

figure 20:

Conceptual axonometric collage reveals the complete interaction of the narratives and the site. The image illustrations how the details extend from the narratives and the hidden moments in order to create an architecture that responds to the historical topography of the plaza. 


\section{CONCLUSION}

Through the analysis of these historical topographical layers there is evidence of forms that exist, that remain undetected, that help to re-read a site's history and its narratives. The notion of moving from a macro to a micro scale reveals these details in a fractal manner, which can help to activate rituals that could be performed within the site. When looking at the chronological history we are often unaware of hidden details within the narratives based on the fact that we associate one event of a history to the previous or a later one in order to establish a sense of order. Being reliant on this association to bridge connections between various events and narratives we overlook the most significant details. Moving from the history to the narratives and into their details a new order begins to reveal itself. It is no longer one that follows a natural chronological order, but rather it follows an intuitive one. Once the details of the narratives are established they can be rearranged intuitively into a new story that responds cohesively to a site's topographical history.

The narratives derive from the history, and as long as these narratives remain within the boundaries of the history in which they are based from they will always lead back to its sources or origins. Meaning, the history is already established and cannot be undone - the events, the moments and narratives have 
SECTION C

already left their mark, but their order is open for interpretation which allows for a new perspective to be created to view these narratives.

Organizing these topographical layers requires a sense of scale, where each individual layer relates to the others proportionately. It is important to maintain this sense of proportion in order for the project to read as a harmonious whole. If the proportions are not connected, a discontinuity can be felt throughout the project, which jeopardizes the ability of the project to adapt itself successfully back into the site and its history. These details and layers should have human scale, which signifies a person's ability to interact with the project. The tactility increases the potential for people to physically engage with the project, successfully transmitting a sensory experience of the narratives onto the people. The idea of scale goes beyond the physical dimensions of a detail, which in fact the objects developed can be produced at any ratio but the word scale in the process of Mikro-Arkitekture also implies materiality. While the tangible scale of an object speaks to the manner in which it physically interacts with the people the material or materials of a detail emphasizes a more tactile quality. The size and materials should be taking into consideration when analyzing the historical topographical layers to best represent the essence of a narrative.

The developed details break away from the standardization of construction components used in everyday building techniques. Mikro-Arkitekture allows for a 
SECTION C

greater connection to be made with the site and its history by developing unique methods of construction. Breaking away from the standardization of construction materials gives greater longevity to a project which the relationship between the past and present is strengthened but it also creates a connection to future narratives. The project is able to thus re-adapt itself based on its adherent connection to an already established history. 


\section{POST-SCRIPT}

Throughout the development of this thesis a variety of goals were set out which a number of them had been achieved while simultaneously generating new questions that developed along the way which can extend the thesis into further investigation. The main objective of this thesis was to bring awareness to a process of designing that follows a more natural and intuitive methodology. It is with the hopes of this process of analyzing the historical aspects of a site that the architecture becomes more in tune with the people, culture and traditions that have shaped the current conditions of a particular site. I believe the thesis to be successful in this manner in portraying how the historical topographical conditioning of a site plays a vital role in its architectural development. Looking at specific narratives of the history allows for an in-depth re-discovery of what is already existing. This in turn allows for the revealing of hidden details that help to enhance or shift ones perspective of a history, but the selection process of the narratives raised questions about which ones should be used in the investigation and why. In the thesis I defend the fact that the selection process is an important conscious act done by the architect but this notion could be further enhanced by providing a series of guidelines as to which narratives to investigate. In the development of the plaza design the narratives used were major events of the history but the investigation could have also included more subtle and minor events that better reflective more day-to-day rituals that appear more natural, rather than particular grandeur events and spectacles that take place once a year. 
The thesis, I believe, was successful in showing a creative methodology that could be successfully implemented in the design process of a project. Utilizing this notion of historical topography and the re-organization of the narratives in order to re-read an already establish history allows for a continuity between historical precedence, existing conditions and future historical development of the site. This raises questions with regard to creating an architecture that has a certain ephemeral quality while remaining permanent. For example if the architecture derives from specific annual events than how does the architecture function within an everyday setting. The thesis lays grounds for this notion but could be extended into further detail in the attempt to explain how the architecture developed really functions within the site and how it may or may not change its' overall context within the immediate community and the city itself. Furthermore the thesis would benefit greatly from a more in-depth analysis and explanation of the sites relation to the immediate architecture around it. I believe that a majority of the initial questions and goals of what is Mikro-Arkitekture and how does it function have been answered to the best capabilities. While certain questions remained unanswered, the thesis thus far provides a solid basis for which the idea of Mikro-Arkitekture can be further developed. 


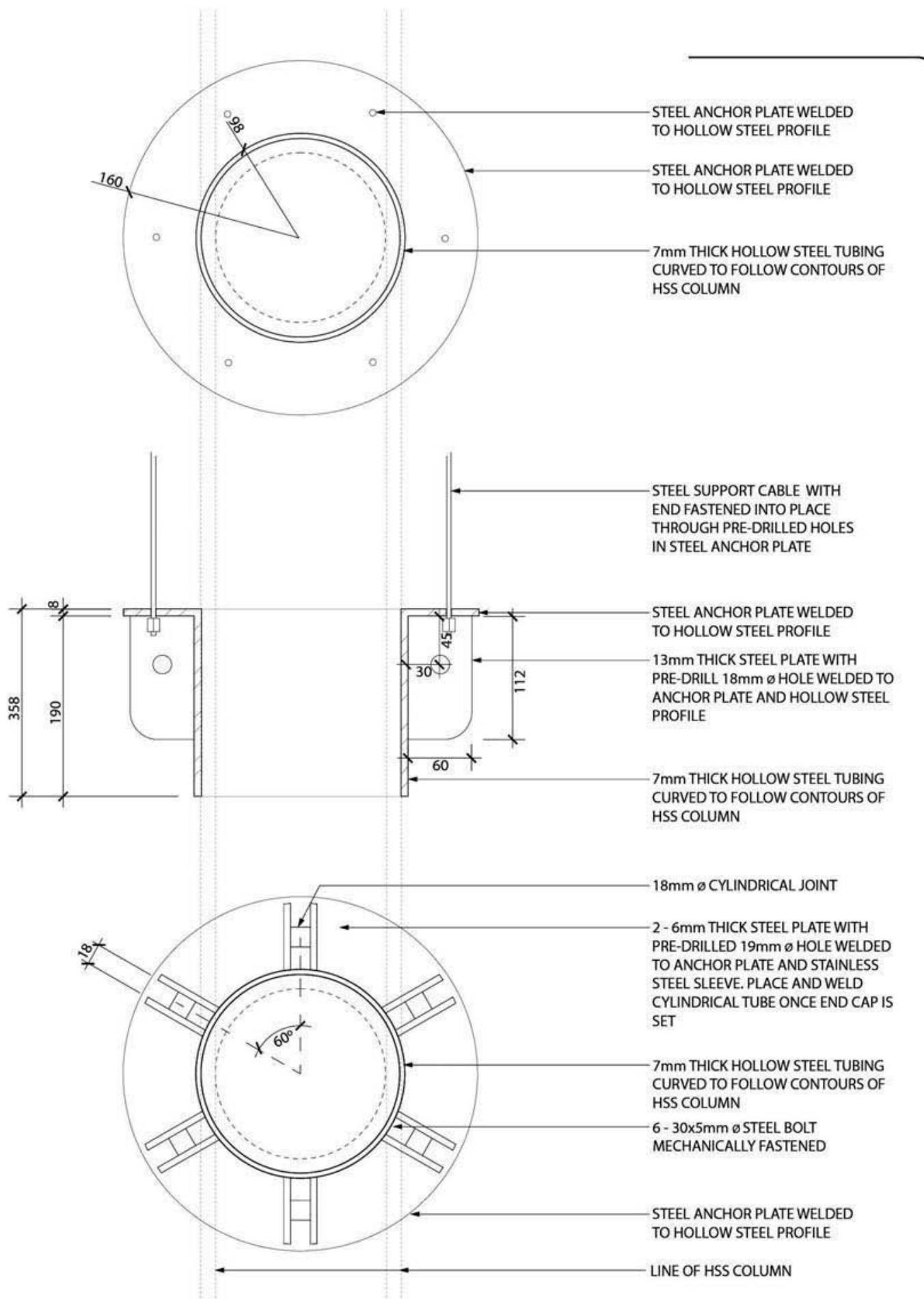



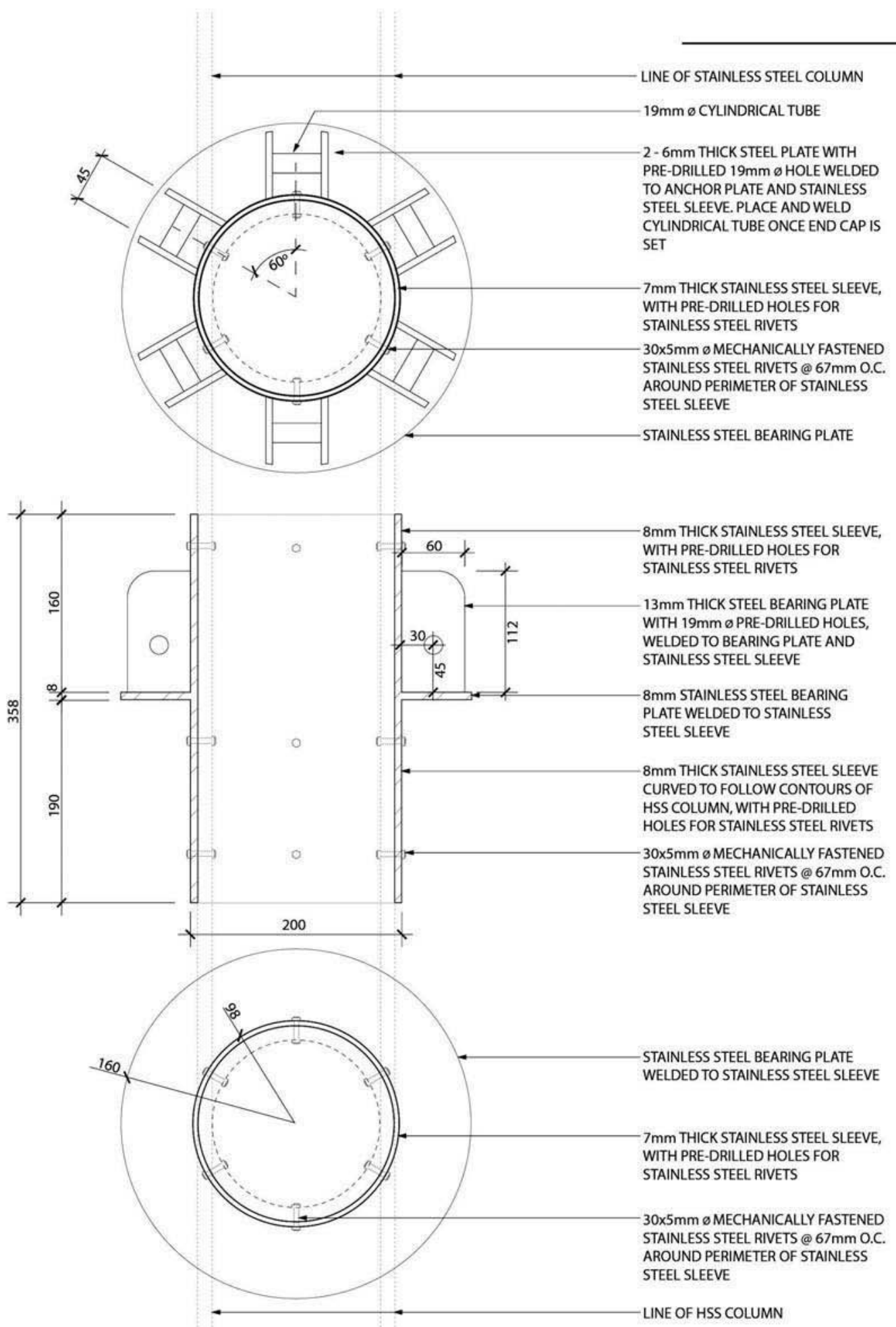

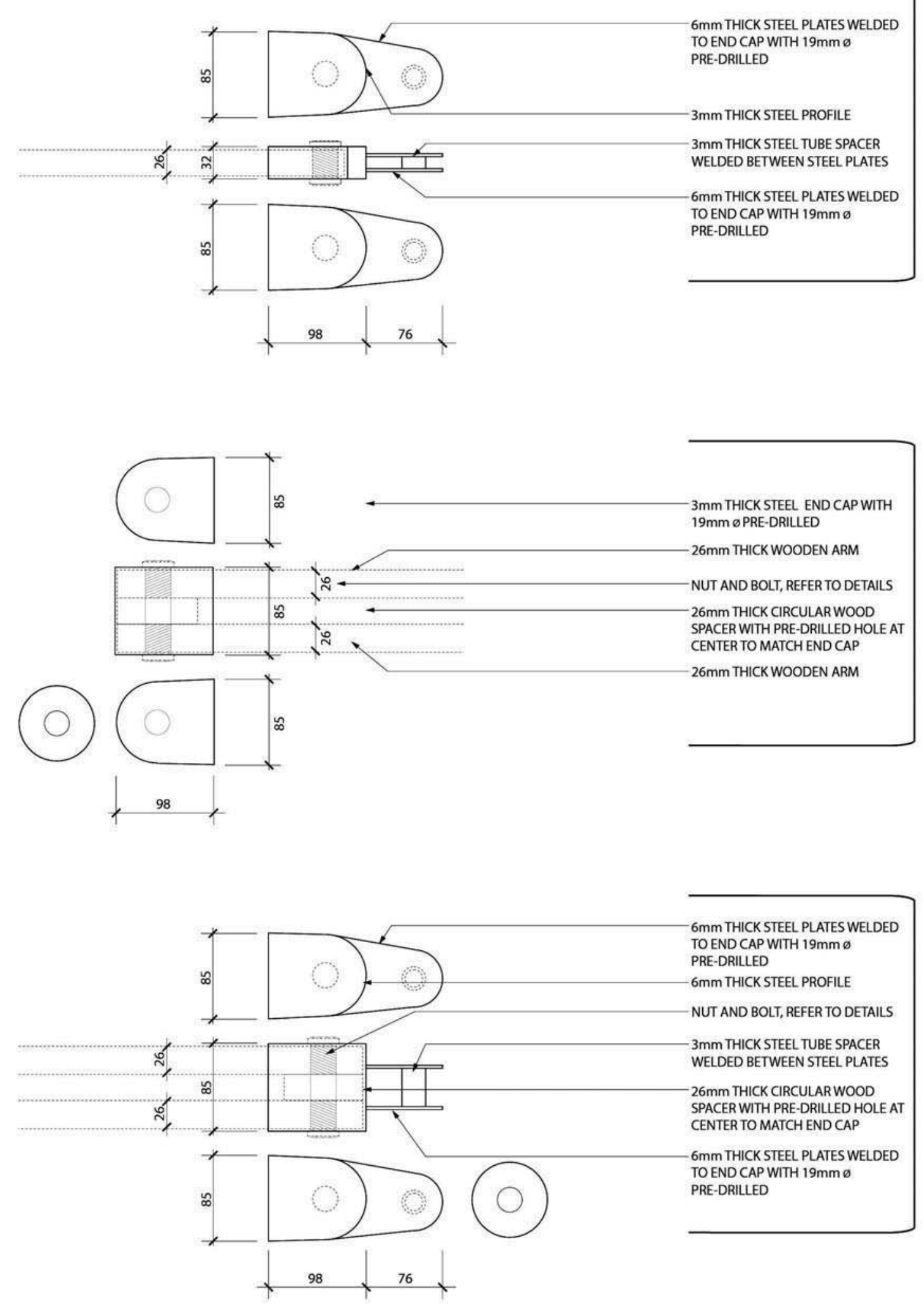
SECTION E

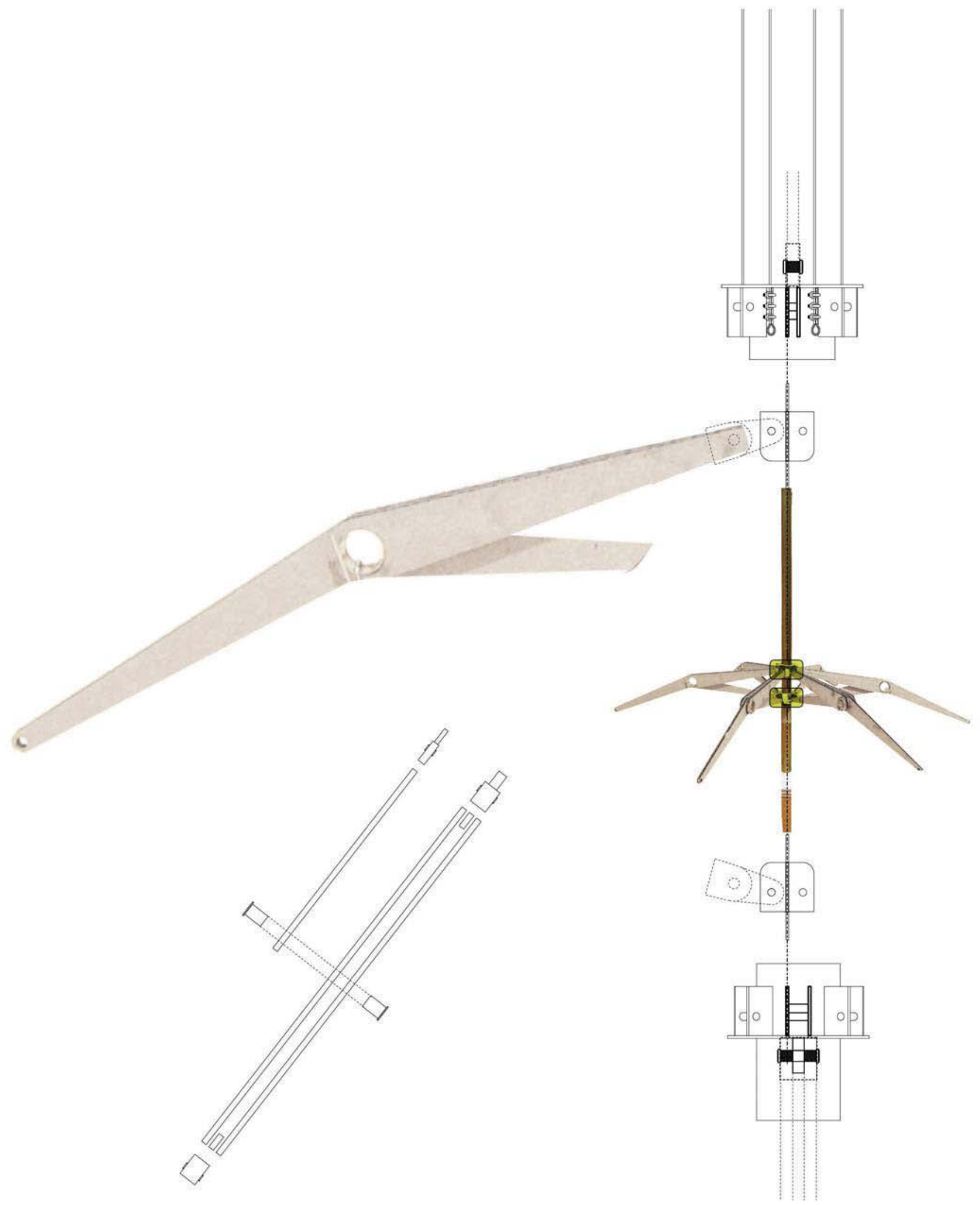


SECTION E

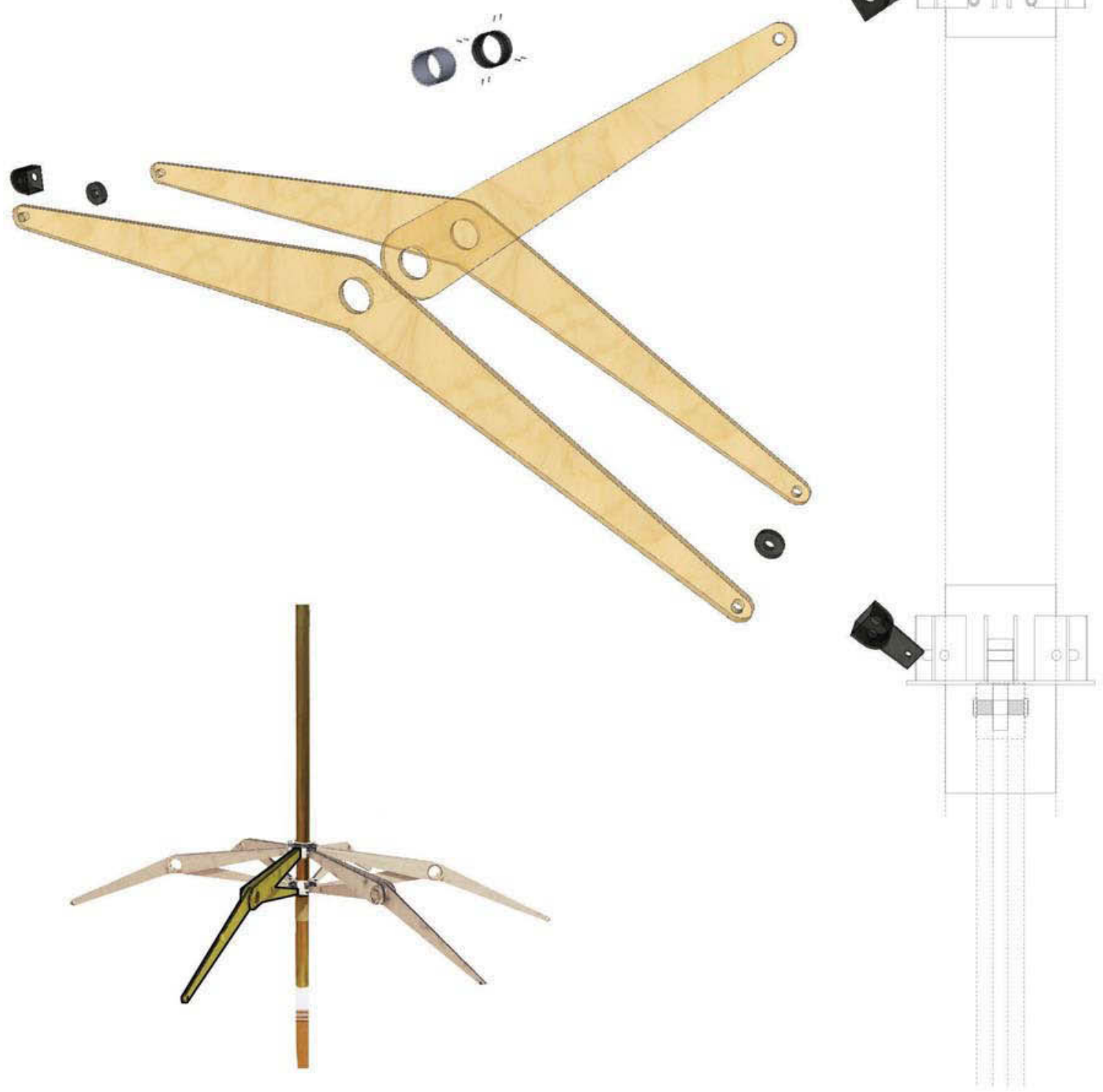




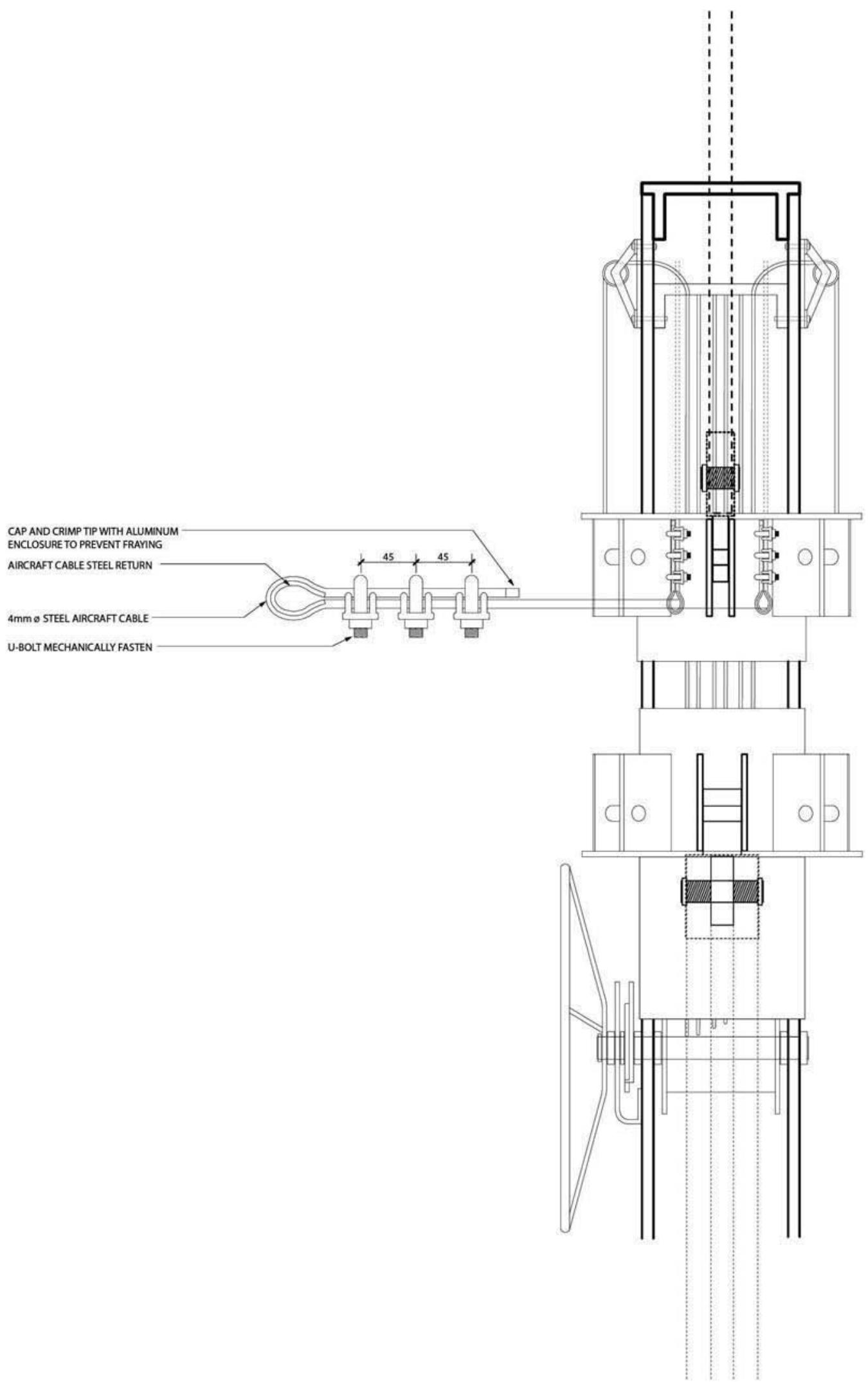




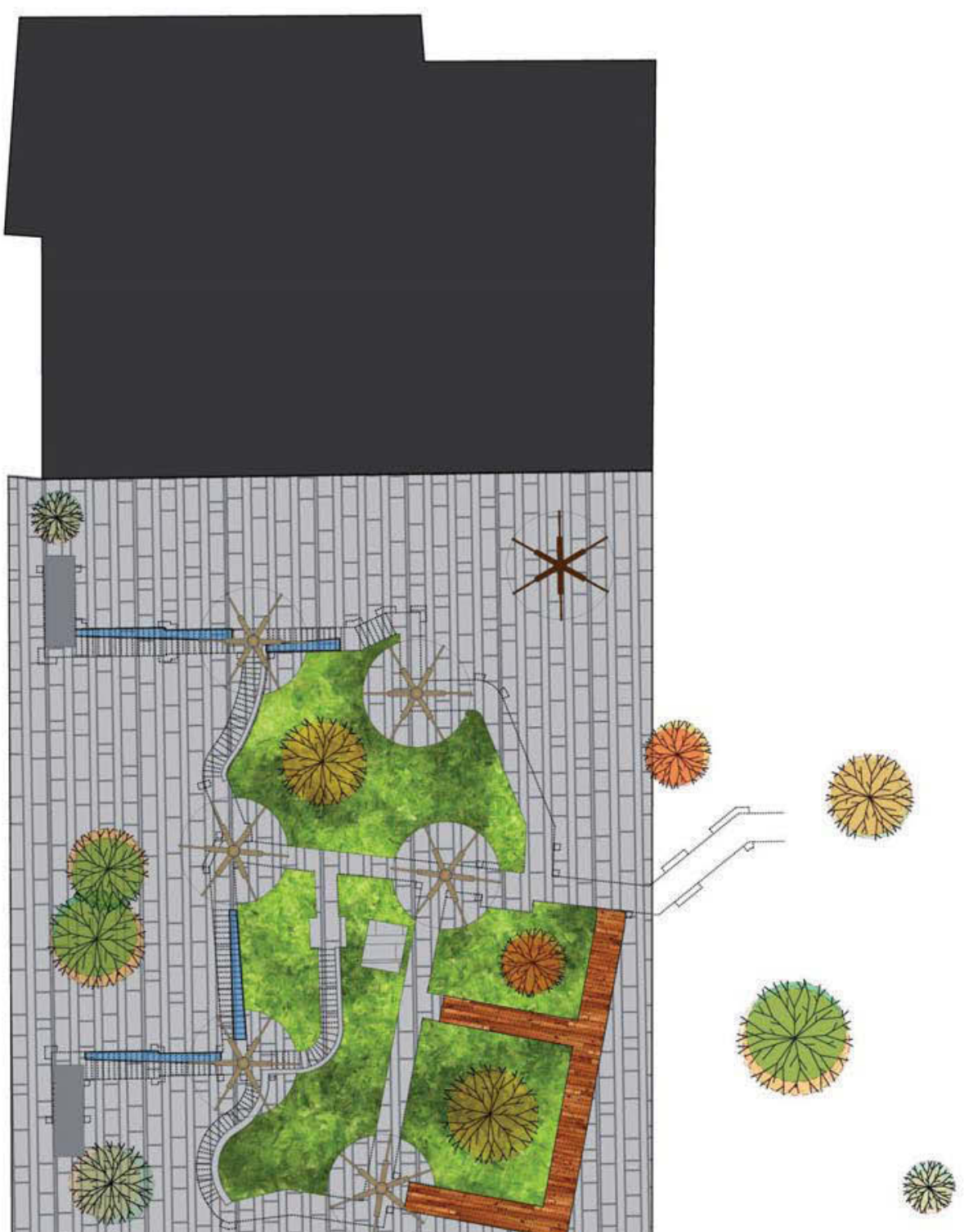


figure 1:

The diagram illustrates the primary steel sleeve that caps the top of the canopy. This sleeve moves vertically up and down controlled by a series of aircraft cables that is secured and threaded into the hollow steel column and attached to a wench device. 6 anchor points allow each arm attach itself to the steel sleeve and to pivot as the angle of the canopy arms change as the sleeve moves up and down.

figure 2:

The diagram illustrates the secondary steel sleeve that remains stationary at midpoint along the hollow steel column. It is bolted and anchored into place and provides 6 anchor points for each canopy arm which allow each arm to pivot as the primary steel sleeve moves vertically.

figure 3:

The diagram shows the end caps of the canopy arms that hold the 3 components together. The top and bottom end caps have extended steel plates that attach to the anchor points of the steel sleeves.

figure 4:

The diagram illustrates the relation between the primary and secondary steel sleeves and the top and bottom end cap that attaches to the steel sleeve. Furthermore it demonstrates the connection and relationship of the canopy arms to the end caps.

figure 5:

The diagram further illustrates this relationship between the micro-details of the various components used to construction a single canopy arm. The various connectors and end caps function as a singular unit and anchor to the primary and secondary steel plate.

figure 6:

The diagram illustrates a hybrid section of the canopy and all the components that allow it to function. This image follows the idea of moving from a macro to a micro details and how each part is in scale and proportion with each other in order to create a unique architecture that responds to specific conditions. The primary and secondary steel sleeves are shown encasing the hollow steel column as the aircraft cable attaches itself to the primary sleeve and then threaded through pre-drilled holes in the column attaching itself to the wench device which is controlled by a wheel. The diagram shows the relationship between the details of the canopy arms and other micro-details that help to animate the architecture. 
SECTION E

figure 7:

The diagram illustrates a final conceptual site plan which shows how the various details such as the glass reveals, concrete formworks, green spaces and canopies. 


\section{PRIMARY BIBLIOGRAPHY}

Bovill, Carl. Fractal Geometry in architecture and design (Boston: Birkhauser, 1996).

Schittich, Christian; edited. Small Structures: compact dwellings, temporary structures, room modules (Switzerland: Birkhauser, 2010).

Coastes, Nigel. Narrative Architecture (London, UK: John Wiley \& Sons ltd, 2012).

Crook, J. Mordaunt. "Architecture and History," Design and Practice in British Architecture: Studies in Architectural History, Vol. 27 (spring 1984), pp. 555-578.

Ginzburg, Carlo. "Microhistory: two or three things I know about it," Critical Inquiry, Vol. 20 No. 1 (autumn, 1993).

Haldane, John Burdon Sanderson. A.R.P. (London, UK: Left Book Club, 1938).

Hopfner, John. "Microarchitecture - experiments in space optimisation," in Small Structures: compact dwellings, temporary structures, room modules (Germany: Edition Detail, 2010).

Kalhofer, Gerhard. "Mobile Architecture," in Small Structures: compact dwellings, temporary structures, room modules (Germany: Edition Detail, 2010).

Leatherbarrow, David. Architecture oriented otherwise (New York, USA: Princeton Architectural Press, 2009).

Leatherbarrow, David. Uncommon ground: architecture, technology, and topography (Cambridge, Massachusetts: MIT Press, 2000).

Miralles, Enric. Mixed talks (London, UK: Academy Editions, 1995).

Miralles, Enric; Tagliabue, Benedetta. El Croquis. (Madrid: El Croquis, 2009).

Picon, Antoine. Ornament: the politics of architecture and subjectivity (Chichester: John Wiley and Sons, Ltd). 
Robinson, Sarah. Nesting: body, dwelling, mind (Richmond, California: William Stout Publishers, 2011).

Temple, Nicholas. Disclosing Horizons: Architecture, Perspective and Redemptive Space (London, UK: Routledge, 2006).

Vazquez Montalban, Manuel. Barcelona (London, UK: Verso, 1992).

Zabalbeascoa, Anatxu. Igualada Cemetery: Enric Miralles and Carme Pinos (London, UK: Phaidon Press, 1996).

\section{SECONDARY BIBLIOGRAPHY}

Alberti, Leon Battista. On the Art of Building in ten books (Cambridge, Massachusetts: MIT Press, 1988).

Dimitris Kottas, Architecture and Construction in Concrete (Links International, Ceg, 2012).

Giralt-Miracle, Daniel. Gaudi, la busqueda de la forma: espacio, geometria estructura y construccion (Barcelona, Catalunya: Lunwerg Editores, 2002).

Gray, Diana. Mies van der Rohe Pavilion Award for European Architecture 1994 (Barcelona: Grup 3, s.a., 1995).

Harris, James. Fractal Architecture: Organic Design Philosophy in Theory and Practice (Albuquerque, USA: University of New Mexico Press, 2012).

Manacorda, Francesco; Yedgar, Ariella. Radical Natural: art and architecture for a changing planet (London, UK: Barbican Art Gallery, 2009).

Marek Heinel and Lisa Rogers, edited, Low Price Houses (Berlin: Braun Publishing AG, 2011). 
Martorell Portas, Vicente; Florensa Ferrer, Adolfo; Martorell Otzet, V. Historia del urbanismo en Barcelona; del plan Cerda al Area Metropolitana (Barcelona, Catalunya: Editorial Labor, 1970).

Matt Bua and Maximilian Goldfarb, Architectural Inventions: Visionary Drawings (London: Laurence King Publishers, 2012).

Pickover, Clifford. Fractal Horizons: the future use of fractals (New York, USA: St. Martin's Press, 1996).

Stanford Anderson, Gail Fenske and David Fixler, edited, Aalto and America (New Haven: Yale University Press, 2012).

William, Blackwell. Geometry in Architecture (Columbus, USA: Key Curriculum Press, 1984). 
SECTION F

\section{LIST OF ILLUSTRATIONS}

Image $1 \quad$ Miralles, Enric. Mixed talks. London, UK: Academy Editions, 1995.

Image 2-6 Le Mortelle sas di Alessia Antinori \& C. AREA. December 2010. Issue 113.

Image $7 \quad$ Miralles, Enric. Mixed talks. London, UK: Academy Editions, 1995.

Image 8-9 Miralles, Enric; Tagliabue, Benedetta. El Croquis. Madrid: El Croquis, 2009.

Image 10-11 Miralles, Enric. Mixed talks. London, UK: Academy Editions, 1995.

Image 12 Altdorfer, Albrecht. Battle of ALexander at Issus. 1529. Alte Pinakothek, Munich. Pinakothek. 03 Aug. 2014 http://www.pinakothek.de/en/ albrecht-altdorfer/battle-issus

Image 13-14 Bovill, Carl. Fractal Geometry in architecture and design. Boston:

Birkhauser, 1996.

Image 15 Mapeso, Dean

Image 16-17 Suzanne. Alambra, Spain. 2012. Artifacts and Fictions. Photography. 27 July 2014.

Image 18-19 Miralles, Enric; Tagliabue, Benedetta. El Croquis. Madrid: El Croquis, 2009.

Image 20 Le Mortelle sas di Alessia Antinori \& C. AREA. December 2010. Issue 113.

Image 21-22 Zabalbeascoa, Anatxu. Igualada Cemetery: Enric Miralles and Carme Pinos. London, UK: Phaidon Press, 1996.

Image 23-25 Le Mortelle sas di Alessia Antinori \& C. AREA. December 2010. Issue 113.

Image 26 Smith, G.L. Guidebook to Stowe. 1773. Stowe, Buckinghamshire, England. 03 Aug. 2014 http://www.fulltable.com/vts/g/gd/18.jpg

Image 27-28 Zabalbeascoa, Anatxu. Igualada Cemetery: Enric Miralles and Carme Pinos. London, UK: Phaidon Press, 1996.

Image 29 Mapeso, Dean 
SECTION F

Image $30 \quad$ Mapeso, Dean 